EPJ manuscript No.

(will be inserted by the editor)

\title{
The pion-nucleon scattering lengths from pionic deuterium *
}

\author{
Ulf-G. Meißner ${ }^{1,2}$, Udit Raha ${ }^{1}$, and Akaki Rusetsky ${ }^{1,3}$ \\ 1 Universität Bonn, Helmholtz-Institut für Strahlen- und Kernphysik (Theorie), \\ Nußallee 14-16, D-53115 Bonn, Germany \\ 2 Forschungszentrum Jülich, Institut für Kenphysik (Theorie), D-52425 Jülich, Germany \\ 3 On leave of absence from: High Energy Physics Institute, Tbilisi State University, \\ University St. 9, 380086 Tbilisi, Georgia \\ Received: date / Revised version: date
}

\begin{abstract}
We use the framework of effective field theories to discuss the determination of the $S$-wave $\pi N$ scattering lengths from the recent high-precision measurements of pionic deuterium observables. The theoretical analysis proceeds in several steps. Initially, the precise value of the pion-deuteron scattering length $a_{\pi d}$ is extracted from the data. Next, $a_{\pi d}$ is related to the $S$-wave $\pi N$ scattering lengths $a_{+}$and $a_{-}$. We discuss the use of this information for constraining the values of these scattering lengths in the full analysis, which also includes the input from the pionic hydrogen energy shift and width measurements, and throughly investigate the accuracy limits for this procedure. In this paper, we also give a detailed comparison to other effective field theory approaches, as well as with the earlier work on the subject, carried out within the potential model and multiple scattering framework.
\end{abstract}

PACS. 36.10.Gv - 12.39.Fe - 13.75.Cs - 13.75.Gx

\section{Introduction}

The Pionic Hydrogen collaboration at PSI [1,2] has performed high precision measurements of the strong interaction shift $\varepsilon_{1 s}$ and width $\Gamma_{1 s}$ of the $1 s$ state of pionic deuterium from the $3 p-1 s X$-ray transition. The (complex) pion-deuteron scattering length was extracted from these measurements with the use of the leading-order Deser formula [3]

$$
-\varepsilon_{1 s}+i \frac{\Gamma_{1 s}}{2}=\frac{4 E_{1 s}}{r_{B}} a_{\pi d},
$$

where $E_{1 s}=\frac{1}{2} \alpha^{2} \mu_{d}$ is the Coulomb binding energy in the $1 s$ state, and $r_{B}=\left(\alpha \mu_{d}\right)^{-1}$ denotes the Bohr radius (in these formulae, $\mu_{d}$ stands for the reduced mass of the $\pi d$ system). The most recent measurement of the pion-deuteron scattering length by the Pionic Hydrogen collaboration at PSI [2] yields

$$
a_{\pi d}=(-0.0261( \pm 0.0005)+i 0.0063( \pm 0.0007)) M_{\pi}^{-1}
$$

Performing experiments to determine $a_{\pi d}$ is usually justified by the possibility of extracting independent information about the $\pi N S$-wave isoscalar $\left(a_{+}\right)$and isovector

* This research is part of the EU Integrated Infrastructure Initiative Hadron Physics Project under contract number RII3CT-2004-506078. Work supported in part by DFG (SFB/TR 16, "Subnuclear Structure of Matter"). $\left(a_{-}\right)$scattering lengths. What makes this enterprise particularly interesting is the fact that in the multiple scattering theory $a_{\pi d}=$ const $\cdot a_{+}+$correction terms. If one could accurately evaluate the higher-order terms in this expression, then a precise measurement of $a_{\pi d}$ would enable one to constrain the value of $a_{+}$, which is in general a rather delicate task. The reason for this is that, since $a_{+}$is much smaller than the isospin-odd scattering length $a_{-}$, a very high accuracy is needed in order to determine $a_{+}$from the measurements of the linear combinations of $a_{+}$and $a_{-}$. This is exactly the case in the experiments measuring the pionic hydrogen energy shift and width, which enable one to determine the combinations $\left(a_{+}+a_{-}\right)$ and $a_{-}$, respectively. Thus, the measurement of $a_{\pi d}$ contributes a complementary piece of information about the scattering lengths, which can be used in the complex theoretical analysis finally aimed at the determination of both $a_{+}$and $a_{-}[4]$. We wish to mention here that these scattering lengths are quantities of fundamental importance in low-energy hadronic physics, since they test the QCD symmetries and the exact pattern of the chiral symmetry breaking. Moreover, since the knowledge of these scattering lengths places a constraint on the $\pi N$ interactions at low energy, it also affects our understanding of more complicated systems where $\pi N$ interaction serves as an input, e.g. $N N$ interaction, $\pi$-nucleus scattering, three-nucleon forces, etc.

Expressing the $\pi d$ scattering length in terms of the parameters characterizing the underlying pion-nucleon and 
Ulf-G. Meißner et al.: The pion-nucleon scattering lengths from pionic deuterium

nucleon-nucleon dynamics is one of the classical problems in conventional nuclear physics based on the potential scattering formalism, see, e.g. [5-13]. Note, however, that the experiments on pionic deuterium will be used to extract the $\pi N$ scattering lengths in QCD and not in any potential model. In other words, using the latter in order to establish the relation between $\pi d$ and $\pi N$ scattering lengths introduces a theoretical error in the analysis of the experimental data, whose magnitude is very hard to control.

In recent years, the problem of a very low energy piondeuteron scattering has been studied within the framework of effective field theories (EFTs). The method originates from the seminal paper of Weinberg [14], where chiral Lagrangians have been systematically applied for the description of interactions of pions with nuclei. In this paper, by using the chiral Lagrangian, one calculates the set of diagrams contributing to the "irreducible transition kernel" for the pion scattering on two nucleons, and the result is then sandwiched between "realistic" deuteron wave functions in order to evaluate the scattering amplitude of the pion on the deuteron. In actual calculations carried out in Ref. [14], the phenomenological deuteron wave function for the Bonn potential model has been used. We wish to note that this last step, in general, can be justified within the framework of the effective field theories, only if the particular process which one is going to describe is dominated by the long-range mechanisms, e.g. by one-pion exchange. On the other hand, when the calculations within such a "hybrid" approach are pursued in higher orders in Chiral Perturbation theory (ChPT), the kernels grow faster with a large momenta and probe shorter distances. Moreover, this short-distance behavior is not necessarily correlated to that of the phenomenological wave function. From this we conclude that in order to obtain a systematic description of the pion-deuteron system, it is preferable to use the deuteron wave functions and the transition kernels, evaluated within the same field-theoretical setting in this case, no specific conjecture about the dominance in the unobservable quantities, like the kernel or the wave function is needed. For the latest work within the hybrid approach, see e.g. [15, 16].

Further development of the approach based on chiral Lagrangians (see, e.g. [15-20]), has followed different paths. In the paper [18] one has used the framework with perturbative pions, whereas the authors of Ref. [19] make use of the so-called Heavy Pion EFT (HP EFT) with the dibaryon field. The latter approach is quite close to the one used in the present work. The technique used in Refs. [18,19] has the advantage that one may easily construct the deuteron wave function in a closed form, since the lowest-order nucleon-nucleon interactions are described by a contact four-nucleon vertex. The central problem in both the papers is related to the calculation of one particular diagram, describing double scattering of pions. These calculations lead to a very strong scale dependence near $\mu \simeq M_{\pi}-$ a natural choice of the scale parameter in this sort of the effective theories. Since, on the other hand, this dependence must be canceled by a contribu- tion from the low-energy constant (LEC), which describes pointlike interactions of four nucleons and two pions, we easily conclude that the magnitude of this LEC can not be small. In the absence of any information about the actual value of this LEC apart from naive dimensional order-ofmagnitude estimates based on the naturalness arguments, one may finally conclude that the theoretical uncertainty in the relation of $\pi d$ and $\pi N$ scattering lengths should be very large.

On the other hand, the results obtained in Ref. [20] seem not to be in agreement with those of Refs. $[18,19]$. The method which is used in Ref. [20], is a systematic extension and elaboration of Weinberg's original proposal, where both the transition kernel and the deuteron wave function are constructed in ChPT (note also, that the systematic derivation of the unitary and the energy-independent potentials within this framework has been discussed recently in Ref. [21]). The approach uses cutoff regularization to deal with the potentials that are growing for a large three-momenta. The typical scales for the cutoff mass $\Lambda$ are somewhat smaller than the hadronic scale in QCD $\sim 1 \mathrm{GeV}$ (depending on the order in ChPT in which the calculations are carried out). The results of the calculations are $\Lambda$-dependent, which is a reminiscent of the scaledependence in the dimensional regularization scheme. The bulk of this $\Lambda$-dependence should be canceled by an analogous dependence in the LECs, and the remainder, which is an artifact of the non-perturbative formalism used, should be of a higher order in ChPT. In Ref. [20], the cutoff dependence of the $\pi d$ scattering length has been studied, with the LECs assumed to vanish. In a remarkable contrast with Refs. $[18,19]$, the $\Lambda$-dependence of the results in Ref. [20] turns out to be very mild, thereby concluding that the LECs must have a weak cutoff dependence. If one could interpret the cutoff dependence as an estimate of the uncertainty of the method, then the results of Ref. [20] would amount to a rather accurate prediction of the $\pi d$ scattering length within the framework of ChPT.

The present situation which was described above is unacceptable from the point of view of both the theory and the phenomenological analysis of the data. From the theoretical point of view, the calculations carried out in Ref. [20], clearly indicate that the diagrams in which the virtual pions are emitted or annihilated, are strongly suppressed. This phenomenon originates from the infrared enhancement of a certain class of the diagrams in the Weinberg scheme, as well as the threshold suppression of the diagrams containing pseudoscalar vertices. In order to accommodate the above feature in the theory with nonperturbative pions, in Ref. [20] a novel counting, inspired by the HP EFT, has been enforced on top of the conventional ChPT Lagrangian. Stated differently, this means that simpler effective theories, which were used in Refs. $[18,19]$, are physically adequate for the problem considered. How can it then be that using a simpler theory, we get an answer which contradicts the answer obtained in Weinberg's framework [20], the very approach one starts from? From the point of view of phenomenology, the existing conflicting predictions, on the one hand, do not en- 
courage the experimentalist's efforts to measure the piondeuteron scattering length to a better accuracy and on the other hand, suggest that the values of the $\pi N$ scattering lengths extracted from the analysis of the $\pi d$ data should be taken with a grain of salt.

The aim of the present paper is to perform a thorough investigation of pion-deuteron scattering at threshold within the framework of low-energy effective theories. In particular, we plan to clearly establish the limits of accuracy for extracting $\pi N$ scattering lengths from the measured $\pi d$ scattering lengths. We also perform a detailed study of the above-mentioned discrepancy between the results obtained within the HP EFT and in the Weinberg approach. Moreover, the investigation of this subtle question, in our opinion, is by itself very informative and sheds light on many peculiar aspects of the effective field theories in general.

The complex problem, which we are going to consider in this paper, naturally falls into several sub-problems, which are characterized by distinct momentum scales. Consequently, instead of trying to describe everything at once, it is convenient to construct a tower of effective field theories, matched one to another, each designed for one particular momentum scale.

i) At the momentum scales $\alpha \mu_{d} \simeq 1 \mathrm{MeV}$, the charged pion and the deuteron form an atom, whose observables are measured by the experiment. The characteristic distances in such an atom - hundreds of $\mathrm{fm}-$ are much larger than the deuteron size, and the binding energy in the ground state, which almost coincides with the Coulomb binding energy $E_{1 s}=\frac{1}{2} \alpha^{2} \mu_{d} \simeq$ $3.5 \cdot 10^{3} \mathrm{eV}$, is much smaller, than the binding energy of the deuteron $\epsilon=2.22457 \mathrm{MeV}$. Stated differently, at these energies the deuteron can not be resolved as a composite particle, and the effective theory, which describes the atom, contains the deuteron field (not the nucleon fields) as an elementary degree of freedom. The hard momentum scale in this effective theory is given by the average value of the threemomentum of the nucleons bound within the deuteron, $\gamma=\sqrt{\epsilon m} \simeq 45 \mathrm{MeV}$, where $m$ stands for the nucleon mass. The expansion parameter in this theory is given by the ratio of the scales $\alpha \mu_{d} / \gamma=O(\alpha)$. The output from the calculations within this effective theory is relation which connects the measured energy shift of the $\pi d$ bound state to the $\pi d$ scattering amplitude at threshold in the next-to-leading order in isospin breaking. In its turn, the latter at the leading order in isospin breaking coincides with the $\pi d$ scattering length $a_{\pi d}$.

ii) Extracting the scattering length from the pionic deuteron one next has to find the relation of this quantity to the $\pi N S$-wave scattering lengths $a_{+}$and $a_{-}$. In order to achieve this goal, we have to construct another effective field theory, in which the independent degrees of freedom are the pion and the nucleon fields, whereas the deuteron emerges as a bound state of the proton and the neutron. The characteristic momentum scale in this theory is defined by the binding momentum $\gamma$. Furthermore, a careful analysis of the re- sults of Ref. [20] provides us with an important clue: The processes, in which the virtual creation and annihilation of pions takes place, are suppressed as compared to the processes where this does not occur (although both processes may formally have the same chiral order). Note that these processes naturally come together in the conventional relativistic QFT. This fact clearly indicates that the most economic way to describe $\pi d$ scattering at threshold is to design an effective field theory, in which the pion creation and annihilation processes are explicitly excluded - all vertices in the Lagrangian contain equal number of ingoing and outgoing pions and nucleons. The whole information about these processes is, however, not lost: it is included in the pertinent LECs of such an effective theory. Moreover, it is also clear that for such small energies, one can treat kinematical relativistic factors as perturbations both for pions and for nucleons.

The calculations of the deuteron properties in the above-described theory, which will be referred to as the heavy-pion effective theory hereafter, dramatically simplify and can be performed analytically. The output of the calculations is the quantity $a_{\pi d}$, expressed in terms of the threshold parameters of the $\pi N$ and $N N$ scattering. The hard scale in such a theory is given by the pion mass $M_{\pi}$, and the expansion parameter is given by the ratio of scales $\gamma / M_{\pi} \simeq 1 / 3$. The matching to the previous effective theory is performed for the $\pi d$ scattering amplitude at threshold: this quantity must be the same in both theories.

Note also that from now on we neglect all isospinbreaking effects (one could not do this at the earlier step, because the pionic deuterium is created predominately by Coulomb interactions.). In this approximation, the threshold scattering amplitude coincides with the $\pi d$ scattering length $a_{\pi d}$. If needed, the isospinbreaking effects can be turned on later.

iii) The simplicity of the calculations in the HP EFT comes at the cost of the large size of the LECs. Since the hard scale of the theory is determined by $M_{\pi}$, this is also the scale that enters in the estimate of the size of the (unknown) LECs in the assumption that these LECs have the natural size (note that some LECs might be parametrically enhanced as compared to the value which is expected on the purely dimensional grounds, see below). On the other hand, if the calculations are done in ChPT, the natural-size LECs are suppressed by a higher scale $4 \pi F_{\pi} \simeq 1 \mathrm{GeV}$ rather than $M_{\pi}$. Thus, the rationale for performing calculations in the Weinberg framework can be formulated as follows. In these calculations, one "resolves" the dynamics of the system at the scales from $M_{\pi}$ up to the scale $\sim 1 \mathrm{GeV}$, which is the energy range where the interactions in the system of few pions and nucleons are predominately determined by (multi)-pion exchanges. One may then assume that the bulk contribution to the HP LECs comes from the momentum region between $M_{\pi}$ and $1 \mathrm{GeV}$ and can be expressed in terms 
of pion loops, which are calculated in the Weinberg scheme.

If we suppose that such a scheme is realized, we arrive at the effective theory, where the characteristic momenta are of order $M_{\pi}$ and the hard scale is given by $4 \pi F_{\pi}$. The expansion parameter, in the absence of other scale, is given by the ratio of two scales $M_{\pi} /\left(4 \pi F_{\pi}\right)$. The matching to the HP EFT is performed for the $S$-matrix element of the process $\pi N N \rightarrow \pi N N$, that determines a particular LEC of the HP Lagrangian. One of the objectives of the present paper is to find out whether doing the calculations in the Weinberg framework and performing the matching to the HP EFT enables one to indeed reduce the uncertainty related to the choice of LECs.

The organization of the paper follows the above-described scheme of "nested" effective field theories. Namely, in section 2 we consider the precise extraction of the $\pi d$ scattering length from the experimental data on the pionic deuterium. Then, in section 3 , we construct the systematic heavy-pion effective theory (HP EFT) in order to calculate the $\pi d$ scattering lengths in terms of the threshold parameters of $\pi N$ and $N N$ interactions. In order to establish the connection to ChPT in the Weinberg scheme, in section 4 we perform the matching of the threshold amplitudes in both theories. We also provide a numerical analysis and discuss the question of accuracy. A detailed comparison to the existing approaches is carried out in section 5. Finally, section 6 contains our conclusions.

\section{Pionic deuterium}

In the experiment at PSI [1,2], one measures the energy of $3 p-1 s X$-ray transition, deducing the strong shift of the pionic deuterium in the $1 s$ state and the $\pi d$ scattering length from this measurement. At the first step, in order to obtain the strong shift, one has to subtract the so-called "electromagnetic shift" from the full measured value, where the former is calculated in the accuracy that matches the experimental precision. At the next step, the $\pi d$ scattering length should be extracted from the strong shift by means of the Deser-type formula (1.1). If required for accuracy considerations, the latter relation can also be generalized to include next-to-leading order isospinbreaking corrections.

To the best of our knowledge, complete calculations of the electromagnetic shift in the pionic deuterium are not available in the literature, except the results contained in table 1 of Ref. [1], where different contributions are given without a derivation. The investigations in Ref. [22] are not complete - as the authors themselves note, they do not include all isospin-breaking corrections at next-to-leading order. In order to have a complete and transparent fieldtheoretical treatment of the pionic deuterium problem at all levels, we find it appropriate here to re-derive the expression for the full energy shift at order $\alpha^{4}, \alpha^{3}\left(m_{d}-m_{u}\right)$, and to check (at least, numerically) the results given in table 1 of Ref. [1].
The method, which will be used in our calculations, is analogous to the one applied recently to describe $\pi^{+} \pi^{-}$ $[23-26], \pi^{-} p[27,28], \pi K[26]$ and $K^{-} p[29]$ atoms. In this section, we display only the final results of the calculations - the necessary details are provided in appendix A. The full binding energy in a given stationary state of the pionic deuterium depends on the principal quantum number $n$, on the orbital quantum number $l$ and on the total angular momentum $j$. For a given $l$ (except $l=0$ ) the total angular momentum $j$ takes the values $j=l-1, l, l+1$. This splitting, which is explicitly evaluated in appendix A, is tiny. The following averaged value is relevant for the analysis of the experimental data

$$
\bar{E}_{n l}=\frac{1}{6(l+1 / 2)} \sum_{j=l-1}^{l+1}(2 j+1) E_{n l j},
$$

Up to the next-to-leading order in isospin breaking, the full energy shift of the $n l$ state can be separated in what is called "electromagnetic" and "strong "parts. In order to simplify the comparison to the existing results, the former is additionally split by hand in different pieces. Finally, at this order one obtains

$$
\begin{aligned}
& \bar{E}_{n l}=E_{n l}^{\mathrm{em}}+\Delta E_{n l}^{\mathrm{str}}, \\
& E_{n l}^{\mathrm{em}}=E_{n l}^{\mathrm{KG}}+\Delta E_{n l}^{\mathrm{rel}}+\Delta E_{n l}^{\mathrm{fin}}+\Delta E_{n l}^{\mathrm{vac}},
\end{aligned}
$$

In the above formula, we have chosen the same naming scheme as in Ref. [1]. Note that in this paper individual contributions are not specified explicitly, so the identification, which is given below and in table 1 , is performed by analogy with the pionic hydrogen case [30]. Our explicit expressions are given below

$$
\begin{aligned}
E_{n l}^{\mathrm{KG}} & =-\frac{\alpha^{2} \mu_{d}}{2 n^{2}}\left(1+\frac{\alpha^{2}}{n^{2}}\left[\frac{2 n}{2 l+1}-\frac{3}{4}\right]\right), \\
\Delta E_{n l}^{\mathrm{rel}} & =\frac{3 \alpha^{4} \mu_{d}^{2}}{8 n^{4}\left(M_{d}+M_{\pi}\right)}\left(\frac{4 n}{l+1 / 2}-3\right) \\
& -\frac{\alpha^{4} \mu_{d}^{3}}{4 M_{d} M_{\pi} n^{4}}\left(-4 n \delta_{l 0}-4+\frac{6 n}{l+1 / 2}\right) \\
\Delta E_{n l}^{\mathrm{fin}} & =\delta_{l 0} \frac{2}{3 n^{3}} \alpha^{4} \mu_{d}^{3}\left(\left\langle r_{d}^{2}\right\rangle+\left\langle r_{\pi}^{2}\right\rangle\right),
\end{aligned}
$$

where $M_{d}$ denotes the mass of the deuteron. An explicit expression for the vacuum polarization contribution is given in Ref. [31], see Eq. (3) of that paper.

In order to be able to compare with the existing results, our numerical calculations have been performed for the same values of the input parameters as in Ref. [1]. We take the deuteron binding energy to be $\epsilon=2.22457 \mathrm{MeV}$, and the charge radii of the deuteron and of the pion are taken to be equal $\left\langle r_{\pi}^{2}\right\rangle^{1 / 2}=0.657 \mathrm{fm}$ and $\left\langle r_{d}^{2}\right\rangle^{1 / 2}=2.106 \mathrm{fm}$, respectively. The calculations were performed for the value of the charged pion mass $M_{\pi}=139.57018 \mathrm{MeV}$. In addition, the calculation of the finite-size correction has been performed by using the latest data for the charge radii 
$\left\langle r_{\pi}^{2}\right\rangle=0.452 \pm 0.013 \mathrm{fm}^{2}[33]$ and $\left\langle r_{d}^{2}\right\rangle^{1 / 2}=2.1303(66) \mathrm{fm}$ see [34] and references therein (the result changes slightly). The results of our calculations and the comparison to the results of Ref. [1] are given in table 1. Note that we have not calculated higher-order (next-to-next-to-leading) isospin-breaking corrections that are given in the last two entries of this table. The results of the calculations from Ref. [1] in these cases should be taken at face value. It can be immediately seen from the table that our calculations completely confirm the results of Ref. [1] at next-toleading order - the agreement between the two columns is perfect.

After having subtracted the calculated electromagnetic contributions from the measured transition energy, one finally arrives at the strong shift, which is related to the $\pi d$ scattering length. Since in the $p$-states the strong shift is proportional to $\alpha^{5}$ and is thus tiny, the measurement of the quantity $E_{3 p}-E_{1 s}$ yields directly the strong shift in the $1 s$-state. In next-to-leading order in isospin breaking, the strong shift for the states with $l=0$ is given by

$$
\begin{aligned}
\Delta E_{n 0}^{\mathrm{str}} & =\varepsilon_{n s}-i \frac{\Gamma_{n s}}{2}=-\frac{\alpha^{3} \mu_{d}^{3}}{2 \pi M_{\pi} n^{3}} \mathcal{T}_{\pi d} \\
& \times\left\{1-\frac{\alpha \mu_{d}^{2}}{4 \pi M_{\pi}} \mathcal{T}_{\pi d}\left(s_{n}(\alpha)+2 \pi i\right)+\delta_{n}^{\mathrm{vac}}\right\}, \\
s_{n}(\alpha) & =2\left(\psi(n)-\psi(1)-\frac{1}{n}+\ln \alpha-\ln n\right), \\
\psi(x) & =\Gamma^{\prime}(x) / \Gamma(x),
\end{aligned}
$$

where the quantity $\mathcal{T}_{\pi d}$ denotes the threshold scattering amplitude in the presence of photons, which is obtained from the conventional amplitude by subtracting all singular contributions at threshold (see [23-29] for more details and definitions). The normalization of this quantity is chosen so that in the absence of the isospin-breaking effects, it reduces to the $\pi d$ scattering length

$$
\mathcal{T}_{\pi d}=4 \pi\left(1+\frac{M_{\pi}}{M_{d}}\right) a_{\pi d}+\cdots
$$

where the ellipses stand for terms vanishing at $\alpha=0$ and $m_{d}=m_{u}$. These terms can be in principle evaluated in ChPT in a systematic manner, in analogy with more simple cases of $\pi N[35,36]$ and $N N[37]$ scattering. Further, the quantity $\delta_{n}^{\mathrm{vac}}=2 \delta \Psi_{n}(0) / \Psi_{n}(0)$ stands for the vacuum polarization correction to the strong shift $\left(\delta \Psi_{n}(0)\right.$ denotes the correction of the Coulomb wave function $\Psi_{n}(0)$ at the origin due to the vacuum polarization effects). This correction was evaluated in Ref. [31] only for the ground state. However, the approach used in this paper can be straightforwardly generalized for the radially excited states. Finally, it is interesting to note that the $n$-dependence of the correction term in Eq. (2.4) is universal, since short-range effects are the same in all atomic states. For this reason, even potential models (see, e.g. [32]) agree with our result in what concerns the difference of the correction terms in the states with a different $n$.

\begin{tabular}{lcc}
\hline $\begin{array}{l}\text { Calculated corrections } \\
\text { to } E_{3 p}-E_{1 s}[\mathrm{eV}]\end{array}$ & Ref. [1] & This work \\
\hline Point nucleus (Klein-Gordon) & 3074.69 & 3074.69 \\
Nuclear and $\pi^{-}$finite size & -0.51 & -0.52 \\
& & $-0.53[33,34]$ \\
Vacuum polarization $\alpha(Z \alpha)$ & 3.72 & 3.72 \\
Relativistic recoil & -0.02 & -0.02 \\
Higher order radiative & 0.04 & - \\
corrections & & - \\
Nuclear polarization & 0.03 & - \\
\hline
\end{tabular}

Table 1. Comparison of the electromagnetic corrections to the $E_{3 p}-E_{1 s}$ transition energies, calculated in Ref. [1] and in the present paper. The second entry for the finite-size effect has been obtained, using the latest experimental data on the charge radii. We did not address the calculation of the last two entries in this table.

To summarize, in this section we have checked the validity of the procedure which is used for the theoretical analysis of the pionic deuterium data at PSI. The calculated electromagnetic shift agrees very well to the one given in Ref. [1]. Further, we have obtained the general expression for the (complex) strong energy shift of the pionic deuterium in the next-to-leading order in isospin breaking, in terms of the $\pi d$ threshold scattering amplitude. This relation should in principle be used to replace the lowest-order formula (1.1) in the data analysis. Note however, that the Coulomb correction which is explicitly displayed in Eq. (2.4) (second term in the brackets), is of order of $2 \cdot 10^{-3}$, if one replaces $\mathcal{T}_{\pi d}$ by Eq. (2.5) and uses the value of the scattering length given in Eq. (1.2). Note also that $10^{-2} \cdots 10^{-3}$ is an expected order of magnitude for the vacuum polarization contribution in Eq. (2.4), see Ref. [31]. Since there are no obvious reasons for having an anomalously large isospin-breaking correction in the quantity $\mathcal{T}_{\pi d}$ either (see e.g. [1,2] and references therein), in the following we do not consider isospin-breaking corrections to the energy shift at all and concentrate on the lowest-order relation Eq. (1.1). If it turns out that the determination of the $\pi N$ scattering lengths from the analysis of the pionic deuterium data can be performed at a few percent level that requires the inclusion of the isospinbreaking effects in Eq. (1.1), one can always go back to the relation Eq. (2.4).

\section{Heavy-pion effective theory for $\pi \mathrm{d}$ scattering}

\subsection{The Lagrangian}

The findings of Ref. [20], as well as the earlier work on the subject (see, e.g. [14]) serve as a clear indication of the fact that the chiral counting is not the most suitable one to be applied for the description of low-energy $\pi d$ scattering. Most straightforwardly, this can be visualized by comparing the contributions from the individual 
diagrams, depicted in Fig. 2 of [20], which is reproduced here, in Fig. 1. The contribution from the diagrams $1 b+1 c$ is by two orders of magnitude smaller than the contribution from 1a, although all three diagrams emerge at the same chiral order. The reason for this difference is that, in contrary to 1a, the diagrams $1 \mathrm{~b}+1 \mathrm{c}$ describe processes with the virtual pion emission/absorption (additional suppression at a small momenta is caused by the presence of the $\gamma_{5}$-vertices in the diagrams $1 \mathrm{~b}$ and $1 \mathrm{c}$ ).

The above discussions lead to the conclusion that it will be convenient to describe the $\pi d$ scattering at threshold in a framework in which the absorption and emission of hadrons does not appear explicitly at the level of Feynman diagrams, but is included in the couplings of the effective Lagrangian. In this manner, we arrive at the theory which must be in a spirit similar to HP EFT (see [19] and references therein). Below we dwell on some differences which exist between the approach used here and in Ref. [19].

i) The HP EFT of Ref. [19] uses the notion of the dibaryon field, whereas we work in terms of the elementary nucleon constituents and sum up all interactions in the $N N$ subsystems. After substituting the expression for the couplings of the Lagrangian in terms of the observables (coefficients in the effective range expansion), the "deuteron propagator" in the present paper coincides with the dibaryon propagator of Ref. [19] in the limit of vanishing effective range. We opt to work in terms of nucleon field in order to make the comparison with ChPT in the Weinberg picture more transparent.

ii) It has been argued that the technique based on the introduction of the dibaryon field enables one to effectively sum up all potentially large contributions coming from the large scattering length and the effective range, whereas higher-order terms can be treated perturbatively. The results of the present paper are obtained under an additional assumption that the effective-range term is small, leading to some technical simplifications. This assumption is, however, not critical - the inclusion of the effective-range term is straightforward in our approach and does not affect the conclusions.

iii) In Ref. [19] the authors have studied one particular diagram that corresponds to the double-scattering contribution the the $\pi d$ scattering length. In this paper, a systematic expansion of the quantity $a_{\pi d}$ is performed in the small parameter $x=\gamma / M_{\pi}$, up to and including terms of order $x$. At this order, there are additional diagrams apart from the one mentioned above.

iv) The most important question is the convergence of the series for the $\pi d$ scattering length. We believe that there are (indirect) indications which testify in favor of the convergence. First of all, at the momenta $\gamma \simeq$ $45 \mathrm{MeV}$, the pionless effective theory gives still a reasonable description of the $N N$ sector. As was mentioned, this fact is in agreement with the observation made in Ref. [20] that the "modified power counting" in the $\pi d$ scattering length works much better than
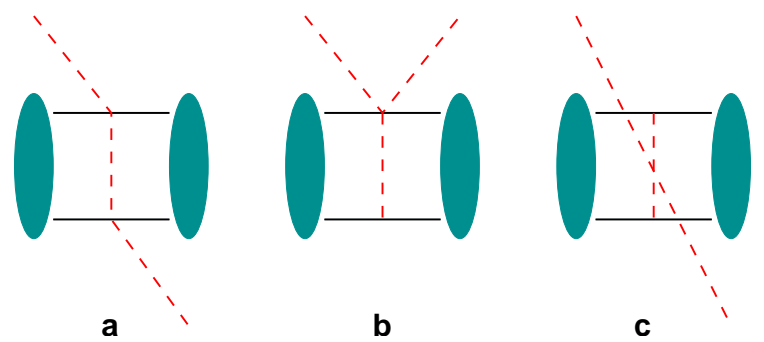

Fig. 1. Leading-order three-body graphs which contribute to the $\pi d$ scattering length in the Weinberg scheme (same as in Ref. [20]). Shaded blobs stand for the deuteron wave function, and the solid and dashed lines denote nucleons and pions, respectively. Numerically, graphs $\mathrm{b}+\mathrm{c}$ are suppressed by two orders of magnitude as compared to the graph a.

the original chiral counting ${ }^{1}$. Yet another justification of the method is provided by the well-known fact that in the Faddeev approach, the multiple-scattering series for the threshold $\pi d$ scattering amplitude are rapidly convergent, since the $\pi N$ scattering lengths are much smaller than the deuteron radius (see e.g. [16] and references therein).

After these preliminary remarks, let us consider the Lagrangian of our theory. By construction, the Lagrangian does contains only vertices with the same number of the incoming and outgoing pions and nucleons. Restricting ourselves to the non-derivative couplings, one may easily write down the most general form of this Lagrangian (in addition, we omit below also all three-body non-derivative terms which contain $\pi_{+}, \pi_{0}$ and/or the $N N$-pair in the ${ }^{1} S_{0}$ state: such terms do not contribute to the $\pi^{-} d$ threshold scattering amplitude at the accuracy we are working)

$$
\begin{aligned}
\mathcal{L} & =\boldsymbol{\pi}^{\dagger}\left(i \partial_{t}-M_{\pi}+\frac{\triangle}{2 M_{\pi}}+\frac{\triangle^{2}}{8 M_{\pi}^{3}}+\cdots\right) \boldsymbol{\pi} \\
& +\psi^{\dagger}\left(i \partial_{t}-m+\frac{\triangle}{2 m}+\frac{\triangle^{2}}{8 m^{3}}+\cdots\right) \psi \\
& +\psi^{\dagger}\left(d_{+}\left(\boldsymbol{\pi}^{\dagger} \boldsymbol{\pi}\right)+i d_{-}\left(\boldsymbol{\pi}^{\dagger} \times \boldsymbol{\pi}\right)\right) \psi \\
& +c_{0}\left(\psi^{T} P_{a} \psi\right)^{\dagger}\left(\psi^{T} P_{a} \psi\right)+c_{1}\left(\psi^{T} P_{i} \psi\right)^{\dagger}\left(\psi^{T} P_{i} \psi\right) \\
& +f_{0}\left(\psi^{T} P_{i} \psi\right)^{\dagger}\left(\psi^{T} P_{i} \psi\right) \pi_{-}^{\dagger} \pi_{-}+\cdots,
\end{aligned}
$$

where the ellipses stand for the omitted three-body terms, as well as for the higher-order terms in the derivative expansion. The non-relativistic pion and nucleon fields are defined as $\boldsymbol{\pi}=\left(\pi_{1}, \pi_{2}, \pi_{3}\right)$, where $\sqrt{2} \pi_{ \pm}=\pi_{1} \mp i \pi_{2}$, $\pi_{0}=\pi_{3}$ and $\psi=\left(\begin{array}{c}p \\ n\end{array}\right)$. Further, $P_{a}$ and $P_{i}$ denote the projection operators onto the ${ }^{1} S_{0}$ and ${ }^{3} S_{1}$ states, respec-

\footnotetext{
${ }^{1}$ And vice versa, one may treat the HP EFT, as the systematic field-theoretical realization of the counting $\gamma \ll M_{\pi} \ll$ $4 \pi F_{\pi}$, which is heuristically implemented in the "modified power counting" of Ref. [20].
} 
tively

$$
P_{a}=\frac{1}{\sqrt{8}} \tau_{2} \tau_{a} \sigma_{2}, \quad P_{i}=\frac{1}{\sqrt{8}} \sigma_{2} \sigma_{i} \tau_{2},
$$

where $\sigma$ and $\tau$ are the Pauli matrices in the spin and isospin space, respectively. Note that we have not introduced an elementary deuteron field in the Lagrangian. In our approach, the deuteron emerges as a bound-state pole in the Green functions after the non-perturbative resummation of the lowest-order four-nucleon vertex.

In the above Lagrangian, $d_{ \pm}, c_{0,1}, f_{0}$ stand for the effective low-energy couplings. These should be determined from matching of the various observables. Using dimensional regularization for calculating the loops enormously simplifies these calculations: as it is well known, all twoparticle bubbles vanish at threshold and the results of the tree-level matching in the two-particle sectors remain intact. For example, the constants $d_{ \pm}$are related to the $\pi N$ scattering lengths through

$$
a_{ \pm}=\frac{m M_{\pi}}{2 \pi\left(m+M_{\pi}\right)} d_{ \pm}
$$

and this relation remains unaffected by loop corrections. As concerning the constant $c_{1}$, we find it more convenient to perform the matching in the ${ }^{3} S_{1}$ channel for the deuteron binding energy, and not for the scattering length in the $n p$ system. The difference between these two methods shows up at higher orders.

\subsection{The deuteron}

In the two-nucleon sector of the HP EFT, there is no trace of pion-nucleon interactions: $N N$ scattering is described completely in terms of contact four-nucleon interactions. The only possible loop diagrams are the $s$-channel bubbles containing the vertices with the couplings $c_{0,1}$. At higher orders, one should also include the derivative four-nucleon vertices.

Consider the following connected four-point function in $D$ dimensions

$$
\begin{aligned}
& (2 \pi)^{D} \delta^{D}\left(p_{1}+p_{2}-q_{1}-q_{2}\right) G_{N}(P ; p, q) \\
= & i^{4} \int d^{D} x_{1} d^{D} x_{2} d^{D} y_{1} d^{D} y_{2} \mathrm{e}^{i p_{1} x_{1}+i p_{2} x_{2}-i q_{1} y_{1}-i q_{2} y_{2}} \\
\times & \left\langle 0\left|T \psi\left(x_{1}\right) \psi\left(x_{2}\right) \psi^{\dagger}\left(y_{1}\right) \psi^{\dagger}\left(y_{2}\right)\right| 0\right\rangle_{c}
\end{aligned}
$$

where all spin and isospin indices have been suppressed, and the CM and relative momenta are defined as

$$
\begin{aligned}
& P=p_{1}+p_{2}=q_{1}+q_{2}, \\
& p=\frac{1}{2}\left(p_{1}-p_{2}\right), \quad q=\frac{1}{2}\left(q_{1}-q_{2}\right) .
\end{aligned}
$$

The center-of-mass (CM) frame corresponds to $P^{\mu}=\left(P^{0}, \mathbf{0}\right)$, and $D \rightarrow 4$ in physical space.
At the energy $P^{0} \rightarrow P_{B}^{0}(\mathbf{P})=M_{d}+\mathbf{P}^{2} / 2 M_{d}$, the four-point function Eq. (3.4) develops a bound-state pole corresponding to the deuteron

$$
G_{N}(P ; p, q) \rightarrow-i \sum_{i=1}^{3} \frac{\Psi_{i}(p) \Psi_{i}^{\dagger}(q)}{P_{B}^{0}(\mathbf{P})-P^{0}}+\text { regular terms }
$$

where the deuteron wave function is defined as

$$
\begin{aligned}
\Psi_{i}(p) & =\int d^{D} x \mathrm{e}^{i p x}\left\langle 0\left|T \psi\left(\frac{x}{2}\right) \psi\left(-\frac{x}{2}\right)\right| B, i\right\rangle, \\
\Psi_{i}^{\dagger}(q) & =\int d^{D} y \mathrm{e}^{-i q y}\left\langle B, i\left|T \psi^{\dagger}\left(\frac{y}{2}\right) \psi^{\dagger}\left(-\frac{y}{2}\right)\right| 0\right\rangle,
\end{aligned}
$$

and the sum in Eq. (3.6) runs over the polarizations of the deuteron spin.

On the other hand, one may evaluate the 4-point function Eq. (3.4) with the use of the Lagrangian Eq. (3.1) that amounts to the resummation of the geometrical series corresponding to the $s$-channel bubbles with four-nucleon vertices. Further, since at the leading order the deuteron is a purely ${ }^{3} S_{1}$ state, we may put $c_{0}=0$ in order to get the deuteron pole. As the result of this resummation, one gets

$$
\begin{aligned}
G_{N}( & P ; p, q)=\sum_{i=1}^{3} \frac{2 P_{i}^{\dagger}}{\left(w\left(\mathbf{p}_{1}\right)-p_{1}^{0}\right)\left(w\left(\mathbf{p}_{2}\right)-p_{2}^{0}\right)} \\
& \times \frac{i c_{1}}{1-c_{1} J\left(P^{0}, \mathbf{P}\right)} \frac{2 P_{i}}{\left(w\left(\mathbf{q}_{1}\right)-q_{1}^{0}\right)\left(w\left(\mathbf{q}_{2}\right)-q_{2}^{0}\right)} \\
& + \text { terms with } c_{0},
\end{aligned}
$$

where, $w(\mathbf{p})=m+\mathbf{p}^{2} / 2 m$, and

$$
\begin{gathered}
J\left(P^{0}, \mathbf{P}\right)=\int \frac{d^{D} l}{(2 \pi)^{D} i} \frac{1}{\left(w(\mathbf{l})-l^{0}\right)\left(w(\mathbf{P}-\mathbf{l})-P^{0}+l^{0}\right)} \\
=\frac{m^{\frac{d}{2}} \Gamma(1-d / 2)}{(4 \pi)^{\frac{d}{2}}}\left(2 m-P^{0}+\frac{\mathbf{P}^{2}}{4 m}-i 0\right)^{\frac{d}{2}-1}, \quad(3.9)
\end{gathered}
$$

with $d=D-1$. In the CM frame $\mathbf{P}=0$ the denominator in Eq. (3.8) develops a pole at $P^{0}=M_{d}=2 m-\epsilon$. This gives (in $d$ dimensions)

$$
1-c_{1} J\left(M_{d}, \mathbf{0}\right)=0, \quad c_{1}=\frac{(4 \pi)^{\frac{d}{2}} \epsilon^{1-\frac{d}{2}}}{m^{\frac{d}{2}} \Gamma(1-d / 2)} .
$$

Finally, in the CM frame the behavior of the Green function near the deuteron pole is given by

$$
\begin{aligned}
& G_{N}(P ; p, q) \rightarrow \sum_{i=1}^{3} \frac{2 P_{i}^{\dagger}}{\left(w\left(\mathbf{p}_{1}\right)-p_{1}^{0}\right)\left(w\left(\mathbf{p}_{2}\right)-p_{2}^{0}\right)} \frac{i Z}{M_{d}-P^{0}} \\
& \quad \times \frac{2 P_{i}}{\left(w\left(\mathbf{q}_{1}\right)-q_{1}^{0}\right)\left(w\left(\mathbf{q}_{2}\right)-q_{2}^{0}\right)}+\text { regular terms, }
\end{aligned}
$$


where the deuteron wave function renormalization constant is given by

$$
Z=\frac{(4 \pi)^{\frac{d}{2}} \epsilon^{2-\frac{d}{2}}}{m^{\frac{d}{2}} \Gamma(2-d / 2)} .
$$

\subsection{Pion-deuteron scattering}

The pion-deuteron scattering amplitude can be extracted from the 6-point connected Green function

$$
\begin{aligned}
& (2 \pi)^{D} \delta^{D}\left(p_{1}+p_{2}+p_{3}-q_{1}-q_{2}-q_{3}\right) G\left(P, Q ; p, q ; p_{3}, q_{3}\right) \\
& =i^{6} \int d^{D} x_{1} d^{D} x_{2} d^{D} x_{3} d^{D} y_{1} d^{D} y_{2} d^{D} y_{3} \\
& \times \mathrm{e}^{i p_{1} x_{1}+i p_{2} x_{2}+i p_{3} x_{3}-i q_{1} y_{1}-i q_{2} y_{2}-i q_{3} y_{3}} \\
& \times\left\langle 0\left|T \psi\left(x_{1}\right) \psi\left(x_{2}\right) \pi_{-}\left(x_{3}\right) \psi^{\dagger}\left(y_{1}\right) \psi^{\dagger}\left(y_{2}\right) \pi_{-}^{\dagger}\left(y_{3}\right)\right| 0\right\rangle_{c},
\end{aligned}
$$

where the $\mathrm{CM}$ and relative momenta of nucleon pairs are again given by Eq. (3.5), and $p_{3}, q_{3}$ denote the pion momenta. Near the mass shell $P^{0} \rightarrow M_{d}+\mathbf{P}^{2} / 2 M_{d}, Q^{0} \rightarrow$ $M_{d}+\mathbf{Q}^{2} / 2 M_{d}$, the six-point function (3.13) develops the double deuteron pole. Since we are interested only in the threshold scattering amplitude, we may take from the beginning $\mathbf{P}=\mathbf{Q}=0$ and $\mathbf{p}_{3}=\mathbf{q}_{3}=0$. Then, in the vicinity of the pole, one has

$$
\begin{aligned}
G\left(P, Q ; p, q ; p_{3}, q_{3}\right) & \rightarrow \sum_{i, j} \frac{i \Psi_{i}(p)}{M_{d}-P^{0}} G_{i j} \frac{i \Psi_{j}^{\dagger}(q)}{M_{d}-Q^{0}} \\
& + \text { regular terms }
\end{aligned}
$$

with

$$
\begin{aligned}
& (2 \pi)^{D} \delta^{D}\left(P+p_{3}-Q-q_{3}\right) G_{i j}=i^{2} \int d^{D} x_{3} d^{D} y_{3} \\
& \times \mathrm{e}^{i p_{3} x_{3}-i q_{3} y_{3}}\left\langle B, i\left|T \pi_{-}\left(x_{3}\right) \pi_{-}^{\dagger}\left(y_{3}\right)\right| B, j\right\rangle .
\end{aligned}
$$

The residue of the quantity $G_{i j}$ on the pion mass shell yields the threshold $\pi d$ scattering amplitude

$$
\lim _{p_{3}^{0}, q_{3}^{0} \rightarrow M_{\pi}} 2 M_{\pi}\left(M_{\pi}-p_{3}^{0}\right)\left(M_{\pi}-q_{3}^{0}\right) G_{i j}=i \delta_{i j} \mathcal{T}_{\pi d} .
$$

On the other hand, in the theory with the Lagrangian Eq. (3.1) the 6-point Green function for vanishing 3-momenta can be given in the following form

$$
\begin{aligned}
& G\left(P, Q ; p, q ; p_{3}, q_{3}\right)=\frac{2 P_{i}^{\dagger}}{\left(m-p_{1}^{0}\right)\left(m-p_{2}^{0}\right)\left(M_{\pi}-p_{3}^{0}\right)} \\
& \times \frac{i(2 \pi)^{D} \delta^{D}\left(P+p_{3}-Q-q_{3}\right) R_{i j}\left(P, Q ; p_{3}, q_{3}\right)}{\left(1-c_{1} J\left(M_{d}, \mathbf{0}\right)\right)^{2}} \\
& \times \frac{2 P_{j}}{\left(m-q_{1}^{0}\right)\left(m-q_{2}^{0}\right)\left(M_{\pi}-q_{3}^{0}\right)}+G_{1} .
\end{aligned}
$$

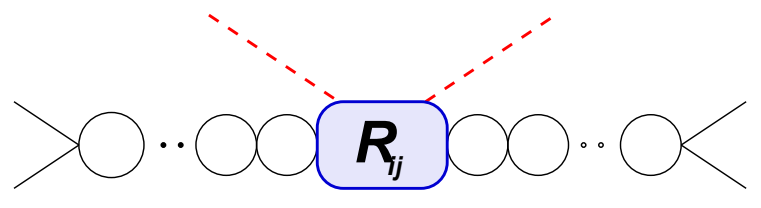

Fig. 2. Definition of the "truncated" Green function $R_{i j}$, Eq. (3.17). The solid and the dashed lines denote nucleons and pions, respectively.

The quantity $R$ in Eq. (3.17) corresponds to the "truncated" Green function, and the factor $\left(1-c_{1} J\left(M_{d}, \mathbf{0}\right)\right)^{-2}$ emerges after the resummation of the $N N$ bubbles in the ${ }^{3} S_{1}$ state before the first and after the last interaction of the pion with one of the nucleons (see Fig. 2). Finally, $G_{1}$ denotes the sum of all diagrams in which the virtual scattering of the pion on one of the nucleons occurs before (or after) all $N N$ interactions, or in which the first (or last) $N N$ interaction happens in the ${ }^{1} S_{0}$ state (the corresponding vertex is proportional to $c_{0}$ ). This class of the diagrams does not develop a double deuteron pole, and contributes only to the regular part of the Green function. Consequently, from the comparison of Eq. (3.17) to Eqs. (3.14), (3.15) and (3.16) one may read of the scattering amplitude at threshold

$$
\delta_{i j} \mathcal{T}_{\pi d}=\left.\mathcal{N} R_{i j}\left(P, Q ; p_{3}, q_{3}\right)\right|_{P^{0}=Q^{0}=M_{d}, p_{3}^{0}=q_{3}^{0}=M_{\pi}}
$$

where

$$
\mathcal{N}=c_{1}^{-2} 2 M_{\pi} Z=\frac{\gamma^{d}}{(4 \pi)^{\frac{d}{2}}} \frac{\Gamma(1-d / 2)}{1-d / 2} 2 M_{\pi} .
$$

Hence, the prescription for calculating the threshold $\pi d$ scattering amplitude is formulated as follows: in the connected 6-point Green function Eq. (3.13) omit all Feynman diagrams, where the very first or very last interaction occurs between the pion and nucleon, or between $N N$ pair in the ${ }^{1} S_{0}$ state. In the remaining diagrams, resum all initial- and final-state $N N$ bubbles and write the final result in a form of Eq. (3.17); read off the quantity $R_{i j}\left(P, Q ; p_{3}, q_{3}\right)$; perform the mass-shell limit, let all 3 momenta vanish, multiply by the normalization factor $\mathcal{N}$, given by Eq. (3.19) and get the threshold scattering amplitude $\mathcal{T}_{\pi d}$.

\subsection{Leading order}

At the lowest order in the expansion parameter $x=\gamma / M_{\pi}$, there is a single contribution to the quantity $R_{i j}$ defined by Eq. (3.17), which is depicted in Fig. 3. At threshold, this contribution equals to

$$
\begin{aligned}
& \left.R_{i j}^{(0)}\right|_{\mathrm{thr}}=4 c_{1}^{2} d_{+} \operatorname{Tr}\left(P_{i}^{\dagger} P_{j}\right) \int \frac{d^{D} l}{(2 \pi)^{D} i} \frac{1}{\left(w(\mathbf{l})-l^{0}\right)^{2}} \\
& \times \frac{1}{w(\mathbf{l})-M_{d}+l^{0}}=\frac{c_{1}^{2} d_{+} m^{\frac{3}{2}} \epsilon^{-\frac{1}{2}}}{4 \pi} \delta_{i j} .
\end{aligned}
$$




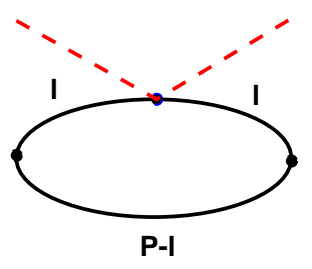

Fig. 3. Leading-order contribution to the $\pi d$ scattering length.

Substituting this result into Eq. (3.18), using Eqs. (2.5), (3.3) and (3.19) and expanding $M_{d}=2 m+O(\epsilon)$, at the leading order we finally obtain

$$
a_{\pi d}^{(0)}=\frac{1+M_{\pi} / m}{1+M_{\pi} / 2 m} 2 a_{+}+O(x),
$$

which of course coincides with the well-known result. Here we only wish to note that our result is valid at all orders in the chiral expansion for the scattering length $a_{+}$. On the other hand, if one works in the Weinberg scheme, one has to identify the contributions to the quantity $a_{+}$order by order in the chiral expansion [20].

\subsection{Next-to-leading order}

Since the quantity $a_{+}$turns out to be very small, the corrections exceed in magnitude the leading-order result. In the HP EFT, four diagrams depicted in Fig. 4 contribute at next-to-leading order. At threshold, we get

$$
\begin{aligned}
R_{i j}^{(1)} & =\delta_{i j} c_{1}^{2}\left(R_{a}+R_{b}+R_{c}+R_{d}\right) \\
R_{a} & =2\left(d_{+}^{2}-2 d_{-}^{2}\right) \int \frac{d^{D} l}{(2 \pi)^{D} i} \frac{d^{D} k}{(2 \pi)^{D} i} \frac{1}{w(\mathbf{l})-l^{0}} \\
& \times \frac{1}{\left(w(\mathbf{l})-M_{d}+l^{0}\right)\left(l^{0}-k^{0}-M_{d}+(\mathbf{l}-\mathbf{k})^{2} / 2 M_{\pi}\right)} \\
& \times \frac{1}{\left(w(\mathbf{k})+k^{0}\right)\left(w(\mathbf{k})-M_{d}-k^{0}\right)}, \\
R_{b} & =2\left(d_{+}^{2}+2 d_{-}^{2}\right) \int \frac{d^{D} l}{(2 \pi)^{D} i} \frac{d^{D} k}{(2 \pi)^{D} i} \frac{1}{w(\mathbf{l})-l^{0}} \\
& \times \frac{1}{\left(w(\mathbf{l})-M_{d}+l^{0}\right)^{2}\left(w(\mathbf{l}-\mathbf{k})-M_{d}-M_{\pi}+l^{0}-k^{0}\right)} \\
& \times \frac{1}{M_{\pi}+k^{0}+\mathbf{k}^{2} / 2 M_{\pi}}, \\
R_{c} & =4 d_{+}^{2} \int \frac{d^{D} l}{(2 \pi)^{D} i} \frac{d^{D} k}{(2 \pi)^{D} i} \frac{d^{D} q}{(2 \pi)^{D} i} \frac{1}{w(\mathbf{l})-l^{0}} \\
& \times \frac{1}{1-c_{1} J\left(M_{d}+M_{\pi}+q^{0},-\mathbf{q}\right)} \frac{1}{M_{\pi}+q^{0}+\mathbf{q}^{2} / 2 M_{\pi}}
\end{aligned}
$$

$$
\begin{aligned}
& \times \frac{1}{w(\mathbf{k}-\mathbf{q})-M_{d}-M_{\pi}+k^{0}-q^{0}} \\
& \times \frac{1}{\left(w(\mathbf{k})-k^{0}\right)\left(w(\mathbf{k})-M_{d}+k^{0}\right)} \\
R_{d} & =\frac{1}{c_{1}^{2}} f_{0} .
\end{aligned}
$$

Note that performing (formally) the limit $M_{\pi} / m \rightarrow 0$ in the quantity $R_{a}$, one gets $1 /\left(l^{0}-k^{0}-M_{d}+(\mathbf{l}-\mathbf{k})^{2} / 2 M_{\pi}\right) \rightarrow$ $2 M_{\pi} /(\mathbf{l}-\mathbf{k})^{2}$. In this limit, it is possible to relate the quantity $R_{a}$ to the average of the operator $1 / \mathbf{q}^{2}$ between the deuteron wave functions in the potential that corresponds to pointlike interaction $c_{1}\left(\psi^{T} P_{i} \psi\right)^{\dagger}\left(\psi^{T} P_{i} \psi\right)$. In the same normalization as in Ref. [20] one obtains

$$
R_{a} \rightarrow 2\left(d_{+}^{2}-2 d_{-}^{2}\right) \frac{2 M_{\pi}}{Z} \frac{1}{(2 \pi)^{3}}\left\langle\frac{1}{\mathbf{q}^{2}}\right\rangle_{\text {w.f. }},
$$

and the standard expression for the double-scattering contribution in the limit $M_{\pi} / m \rightarrow 0$ (see e.g. [20]) is reproduced.

The counting of the above diagrams proceeds as follows. According to Eq. (3.10), the coupling $c_{1}$ counts like $x^{-1}$, and the couplings $d_{ \pm}$count like $x^{0}$. Further, after integrating over the time-like components $l^{0}, k^{0}, q^{0}$, one may rescale $\mathbf{l} \rightarrow \gamma \mathbf{l}, \mathbf{k} \rightarrow \gamma \mathbf{k}, \mathbf{q} \rightarrow \gamma \mathbf{q}$, with $\gamma=O(x)$. Each propagator of a pion or a nucleon counts as $\gamma^{-2} \sim x^{-2}$ and the "virtual deuteron propagator" $c_{1} /\left(1-c_{1} J\right)$ counts as $c_{1} \sim x^{-1}$. With these counting rules, it is straightforward to ensure that $R_{a} \sim R_{b} \sim R_{c}=O\left(x^{0}\right)$ (modulo logarithms). Furthermore, since the constant $f_{0}$ cancels the ultraviolet divergences in the diagrams $4 \mathrm{a}, \mathrm{b}, \mathrm{c}$, it must count at the same order in $x$. This fixes $f_{0}=O\left(x^{-2}\right)$. Note also that the contributions proportional to the coupling constant $c_{0}\left(n n \pi^{0}\right.$ intermediate states $)$ have been dropped from $R_{c}$ altogether at this order. This is related to the orthogonality of the projectors $P_{i}$ and $P_{a}$ given in Eq. (3.2).

The fact that the quantity $R_{c}$ in Eq. (3.22) is proportional to $a_{+}^{2}$ simplifies the calculations considerably. Since $a_{+}$is very small, we shall systematically neglect $a_{+}^{2}$ in all expressions, and thus assume $R_{c}=0$. Evaluating the remaining integrals in dimensional regularization and carrying out the renormalization in a standard manner, we finally arrive at the following expression for the $\pi d$ scattering length at the next-to-leading order

$$
\begin{aligned}
a_{\pi d}^{(1)} & =-\frac{m\left(1+M_{\pi} / m\right)^{2}}{\pi\left(1+M_{\pi} / 2 m\right)} x a_{-}^{2}\left[\left(x_{a}-x_{b}\right) \ln \frac{m \epsilon}{\mu^{2}}\right. \\
& \left.+\left(J_{a}-J_{b}\right)\right]+\frac{M_{\pi}^{4}}{4 \pi^{2}\left(1+M_{\pi} / 2 m\right)} x^{3} f_{0}^{r}(\mu) \\
& +O\left(x^{2}\right)
\end{aligned}
$$

where $f_{0}^{r}(\mu)$ denotes the renormalized coupling constant

$$
f_{0}=\frac{64 \pi^{3}\left(1+M_{\pi} / m\right)^{2}}{\epsilon M_{\pi}^{2}} a_{-}^{2}\left(x_{a}-x_{b}\right) \lambda+f_{0}^{r}(\mu),
$$



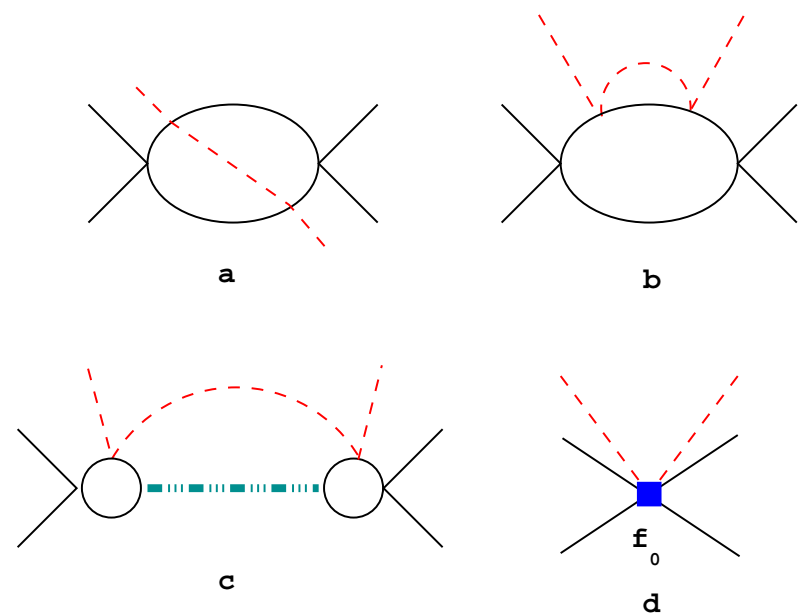

Fig. 4. Next-to-leading order contributions to the $\pi d$ scattering length, see Eq. (3.22): (a) the standard double-scattering contribution, (b) rescattering on a single nucleon, (c) pion rescattering on the infinite chain of the nucleon bubbles ("virtual deuteron", denoted by a thick dot-dashed line), (d) counterterm contribution.

$$
\lambda=\frac{\mu^{2(D-4)}}{16 \pi^{2}}\left(\frac{1}{D-4}-\Gamma^{\prime}(1)-\ln 4 \pi\right),
$$

and $\mu$ denotes the scale of dimensional regularization. Further, $x_{a, b}, J_{a, b}$ denote the integrals over Feynman parameters which depend on the dimensionless variable $M_{\pi} / m$ and emerge from $R_{a, b}$. These integrals are evaluated in appendix B. Here, we only give their approximate values

$$
\begin{array}{ll}
x_{a}=-1.2569, & J_{a}=-1.1334 \\
x_{b}=0.5098, & J_{b}=-0.3731 .
\end{array}
$$

At present, the numerical value of the counterterm $f_{0}^{r}(\mu)$ is not known. For this reason, one has to include this unknown quantity completely in the theoretical error and to estimate the uncertainty that emerges already at next-toleading order. Most easily, this can be done by using the renormalization group equation for the scale dependence of $f_{0}^{r}(\mu)$, which at this order reads

$$
\mu \frac{d}{d \mu} f_{0}^{r}(\mu)=-\frac{8 \pi\left(1+M_{\pi} / m\right)^{2}}{\epsilon M_{\pi}^{2}} a_{-}^{2}\left(x_{a}-x_{b}\right) .
$$

We use the following procedure to estimate the uncertainty. Since the hard scale of the theory is of order of $M_{\pi}$, we may set $f_{0}^{r}(\mu)=0$ at a some scale $\mu \simeq M_{\pi}$ and study the $\mu$-dependence of the plot in the $\left(a_{+}, a_{-}\right)$-plane, which emerges from Eq. (3.24) at a given (experimental) value of $a_{\pi d}$. This plot is given in Fig. 5. Varying $\mu$ in a "reasonable" range, we may thus visualize the error that is caused by the scale dependence. Here, we wish to note that the scale dependence is of course not the only possible source of the theoretical uncertainty in general. In order to have a reliable estimate of the error (in the case of the weak scale dependence) one has, in addition, to use dimensional arguments to estimate the size of the LECs.

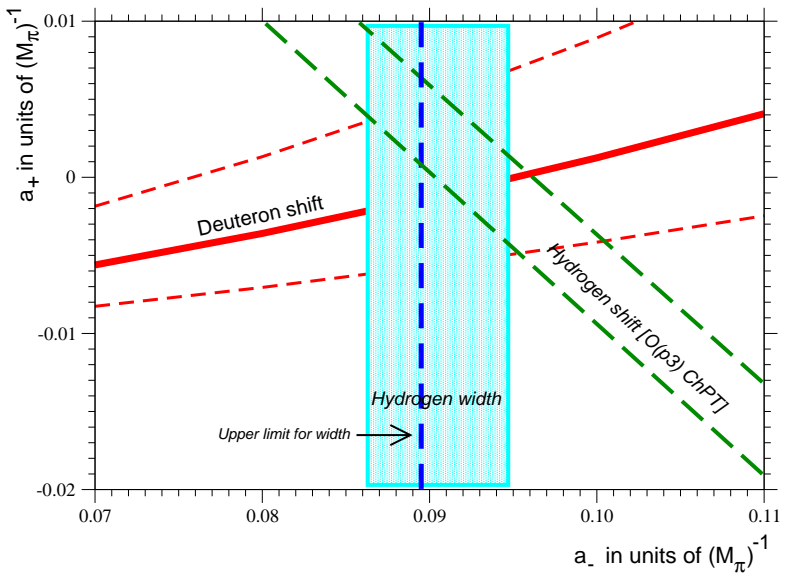

Fig. 5. The constraints on the $S$-wave $\pi N$ scattering lengths $a_{+}$and $a_{-}$from the pionic deuterium energy shift for different values of the scale parameter $\mu$. The central line is given for $\mu=146 \mathrm{MeV}$. The upper and lower bounds correspond to $\mu=250 \mathrm{MeV}$ and $\mu=100 \mathrm{MeV}$, respectively. In addition, we display the constraints from the most recent measurement of the pionic hydrogen shift and width [38]. The upper limit for the width is measured in the $4 p-1 s$ transition in pionic hydrogen.

However, in the case of a strong scale dependence, as in the example considered here, additional arguments are not needed.

The results which are displayed in Fig. 5 are in a qualitative agreement with the findings of Refs. $[18,19]$. Note that these results are obtained at the next-to-leading order in HP EFT. It is unlikely that taking into account higher-order terms will reduce the uncertainty due to the scale dependence. On the contrary, from the comparison with e.g. Eq. (18) of Ref. [19], one may conclude that numerically the most important corrections due to the effective range at this order amount to the multiplication of the loops by the deuteron wave-function renormalization factor $Z_{d}=\left(1-\gamma r_{d}\right)^{-1} \simeq 1.7$, where $r_{d}=1.765 \mathrm{fm}$ is the parameter related to the effective range in the ${ }^{3} S_{1}$ channel. This effect leads to further amplification of the ambiguity related to the scale dependence.

The interpretation of the results displayed in Fig. 5 is unambiguous. The experimental data together with the above theoretical analysis constrain the $S$-wave $\pi N$ scattering lengths within the band which - for large values of $\mu$-also intersects with the common area of two other bands, obtained from the data on the pionic hydrogen energy shift and width. However, without having in advance estimated the numerical value of $f_{0}$, one can not use the measured value of $a_{\pi d}$ for a precise determination of the scattering length $a_{+}$, that was an original motivation for studying the pion-deuteron system. Moreover, since the constant $f_{0}$ parameterizes the short-range physics, it can be only fixed either by using other experimental data (dif- 
ferent from the $\pi d$ scattering process considered here) or through the lattice simulations.

We wish to add that, in order to be consistent with power-counting, the magnitude of the LEC $f_{0}^{r}(\mu)$ is parametrically enhanced by a factor $1 / x^{2} \simeq 10$ as compared to the dimensional estimate $f_{0}^{r}(\mu)=$ const $/ M_{\pi}^{5}$. Numerically, substituting the dimensional estimate into Eq. (3.22), one gets the uncertainty $\sim 3 \%$ in the quantity $a_{\pi d}$, which by far underestimates the actual uncertainty displayed in Fig. 5.

\subsection{Imaginary part}

In the presence of the absorptive channels, the coupling constant $f_{0}$ is not real (note that in order to simplify the notations, we have up to here always omitted the symbol "Re" in this coupling constant, as well as in $a_{\pi d}$ and other quantities, if this does not lead to the confusion). The imaginary part of the $\pi d$ scattering length is given by (cf. with Eq. (3.22))

$$
\operatorname{Im} a_{\pi d}=\frac{\mu_{d}}{2 \pi} \Phi_{0}^{2} \operatorname{Im} f_{0},
$$

where $\Phi_{0}^{2}=\gamma^{3} / 2 \pi$ denotes the square of the deuteron wave function at the origin. Further, the imaginary part of the constant $f_{0}$ is directly related to the inelastic channels, which are "shielded" when constructing the HP EFT. Since we have neglected all electromagnetic effects from the beginning, the bulk contribution to $\operatorname{Im} f_{0}$ is provided by the two-neutron intermediate state, whereas the contribution from the $\gamma n n$ intermediate state is omitted. To take this fact into account, we add the superscript "str" to the pertinent scattering length. To get the imaginary part, which also includes the effect of the $\gamma n n$ state, one writes $\operatorname{Im} a_{\pi d}=\operatorname{Im} a_{\pi d}^{\text {str }}\left(1+1 / R_{\gamma}\right)$, where the experimental value of the quantity $R_{\gamma}=2.83$, which stands for the ratio of the cross-sections of the $\pi^{-} d$ transition into the $n n$ and $\gamma n n$ final states (the Panofsky ratio) and is treated at face value. At the lowest order we get

$$
\begin{aligned}
\operatorname{Im} f_{0}^{\text {str }} & =\pi \int \frac{d^{3} \mathbf{k}_{1}}{(2 \pi)^{3}} \frac{d^{3} \mathbf{k}_{2}}{(2 \pi)^{3}}\left|T\left(p n \pi^{-} \rightarrow n n\right)\right|^{2} \\
& \times(2 \pi)^{3} \delta^{4}\left(p_{1}+p_{2}+p_{3}-q_{1}-q_{2}\right),
\end{aligned}
$$

where $T\left(p n \pi^{-} \rightarrow n n\right)$ stands for the transition matrix element for the process $p\left(p_{1}\right)+n\left(p_{2}\right)+\pi^{-}\left(p_{3}\right) \rightarrow$ $n\left(k_{1}\right)+n\left(k_{2}\right)$, and the above integral has to be evaluated at threshold $\mathbf{p}_{1}=\mathbf{p}_{2}=\mathbf{p}_{3} \rightarrow 0$. After straightforward calculation we get

$$
\begin{aligned}
& \operatorname{Im} f_{0}^{\text {str }}=\frac{m p^{\star}}{4 \pi}\left|T\left(p n \pi^{-} \rightarrow n n\right)\right|^{2}, \\
& \left|\mathbf{k}_{1}\right|=\left|\mathbf{k}_{2}\right|=p^{\star}=\sqrt{m M_{\pi}}+\cdots,
\end{aligned}
$$

where the ellipses stand for the relativistic corrections. Now we note that the above result is compatible with the well-known relation in terms of the $\pi^{-} d \rightarrow n n$ inelastic cross-section at threshold

$$
\begin{aligned}
& \operatorname{Im} a_{\pi d}^{\text {str }}=\frac{1}{4 \pi} \lim _{\left|\mathbf{p}_{3}\right| \rightarrow 0}\left|\mathbf{p}_{3}\right| \sigma\left(\pi^{-} d \rightarrow n n\right), \\
& \sigma\left(\pi^{-} d \rightarrow n n\right)=\frac{m \mu_{d} p^{\star}}{2 \pi\left|\mathbf{p}_{3}\right|}\left|T\left(\pi^{-} d \rightarrow n n\right)\right|^{2},
\end{aligned}
$$

if the transition amplitudes for the processes $\pi^{-} d \rightarrow n n$ and $p n \pi^{-} \rightarrow n n$ are related by

$$
T\left(\pi^{-} d \rightarrow n n\right)=\Phi_{0} T\left(p n \pi^{-} \rightarrow n n\right) .
$$

The equation (3.32) is nothing but the leading-order Deser formula, which is obtained with the assumption that the deuteron radius is much larger than the distances relevant for the interactions in the $\pi N N-N N$ system. The corrections to this formula would then emerge at $O(x)$ relative to the leading-order result. Note also that Eq. (3.32) agrees with the result of [13], obtained within the potential scattering theory, that serves as a good check for the validity of HP EFT.

\section{Matching to ChPT and numerical analysis}

\subsection{Threshold amplitude in the HP EFT}

As it was demonstrated in the previous section, within the HP EFT it is not possible to get an accurate description of the $\pi d$ scattering length in terms of the $S$-wave $\pi N$ scattering length only. The relation between these quantities contains a large unknown short-range contribution (three-body force in the language of the potential scattering theory), which is parameterized through the LEC $f_{0}$.

In this section we shall address the issue whether it is possible to achieve an increased accuracy if one treats the same problem within the Weinberg approach $[14,20]$. In the HP EFT (which is the effective theory of ChPT in the Weinberg picture for the momenta $p \ll M_{\pi}$ ), the LECs (including $f_{0}$ ) receive contributions from two different momentum regions: $M_{\pi}<p<\Lambda$ and $p>\Lambda$ (here $\Lambda \simeq 4 \pi F_{\pi}$ denotes the cutoff mass used in the Weinberg formulation, which is of order of the hard scale in ChPT). In the Weinberg framework, one has "resolved" the momenta at the scales $M_{\pi}<p<\Lambda$. The unknown dynamics at the momenta $p>\Lambda$ is parameterized by new LECs which are now defined at the scale $\Lambda$ instead of $M_{\pi}$. On dimensional grounds, the natural size of these new LECs must be much smaller than the size of old LECs, since $M_{\pi} \ll \Lambda$. Stated differently, if we start from the LECs in the theory at a scale $\Lambda$ and calculate the LECs of the HP EFT in the limit of a large $M_{\pi}$, we must see that the LECs of the HP EFT must be enhanced by the pion loops where the loop momentum runs within $M_{\pi}<p<\Lambda$. It is natural to assume that this momentum region contributes the bulk of the total magnitude of the LEC in question. Could one then separate the large but potentially calculable pion exchange 
contribution to $f_{0}$ from unknown short-range contribution at a scale $\Lambda$ ?

A natural choice of the $S$-matrix element in the scattering sector, which can be used to determine the constant $f_{0}$ through the matching procedure to the Weinberg framework, is that of the elastic process $\pi^{-}(p n)_{3} S_{1} \rightarrow$ $\pi^{-}(p n)^{3} S_{1}$. The matching condition has the form

$$
\begin{aligned}
& T_{W}\left(p_{1} p_{2} p_{3} ; q_{1} q_{2} q_{3}\right)=\prod_{i=1}^{3}\left[2 E_{i}\left(\mathbf{p}_{i}\right)\right]^{1 / 2}\left[2 E_{i}\left(\mathbf{q}_{i}\right)\right]^{1 / 2} \\
& \quad \times T\left(p_{1} p_{2} p_{3} ; q_{1} q_{2} q_{3}\right),
\end{aligned}
$$

where $T$ and $T_{W}$ denote pertinent scattering matrix elements in the HP EFT and in ChPT, respectively. Further, $E_{i}(\mathbf{l})=\sqrt{m^{2}+\mathbf{l}^{2}}, i=1,2$ and $E_{3}(\mathbf{l})=\sqrt{M_{\pi}^{2}+\mathbf{l}^{2}}$ are the relativistic energies of a nucleon and a pion. Note that the kinematical factor in Eq. (4.1) is introduced in order to take into account the different normalization of the oneparticle states in HP EFT and in the Weinberg approach.

We found it convenient to perform matching for the one-particle irreducible (1PI) matrix elements separately in two- and three-particle subsystems (with respect to the non-relativistic pion and nucleon propagators). In the three-particle sector, the scattering amplitude turns out to be singular at threshold: one has to choose the particular kinematics in order to approach the the zero-momentum limit. A possible choice of the external momenta is given by

$$
\mathbf{p}=\mathbf{q}=0, \mathbf{p}_{3}=-\mathbf{P}, \mathbf{q}_{3}=-\mathbf{Q}, \mathbf{P}=-\mathbf{Q} \rightarrow 0,
$$

where the definition of the $\mathrm{CM}$ and the relative momenta is given in Eq. (3.5).

Below, we schematically describe the matching for the above scattering matrix element. Explicit expressions will not be used in the discussion. For our purposes, we only need to demonstrate that such a matching can be performed in principle.

We start with the evaluation of the scattering amplitude $T$ that enters the right-hand side of Eq. (4.1). This quantity receives many contributions (some of them are depicted in Fig. 6). Out of these contributions, the diagrams $6 \mathrm{a}$ and $6 \mathrm{c} 1$ are one-particle reducible and will be excluded from matching. Some of the contributions are singular at threshold. For example, the diagram in Fig. 6e2 contains the factor $|\mathbf{P}|^{-1}$, and the diagrams in Fig. 6e3,e4 are logarithmically singular at small momenta. The threeparticle threshold scattering amplitude $\mathcal{A}(\mu)$, by definition, is obtained from the 1PI matrix element, by subtracting first all singular contributions at $|\mathbf{P}| \rightarrow 0$. Namely, in the vicinity of threshold, the sum of the 1PI diagrams shown in Fig. 6 has the form

$$
T^{(1 \mathrm{PI})}=\frac{T_{-1}}{|\mathbf{P}|}+T_{0} \ln \frac{|\mathbf{P}|}{\mu}+\mathcal{A}(\mu)+O(|\mathbf{P}|),
$$

where the arbitrary scale that enters the logarithm is set equal to the scale of dimensional regularization $\mu$, for simplicity. Further, the quantity $\mathcal{A}(\mu)=f_{0}^{r}(\mu)+\overline{\mathcal{A}(\mu)}$ contains additive contribution from the LEC $f_{0}^{r}(\mu)$, which

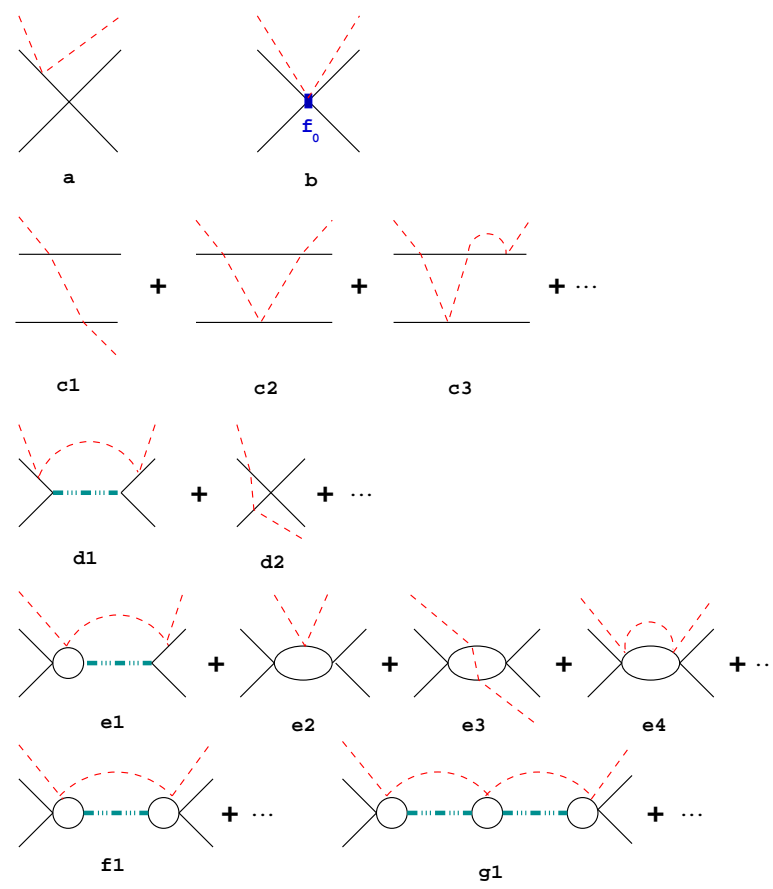

Fig. 6. Lowest-order diagrams contributing to the threshold scattering amplitude for the process $\pi^{-}(p n)_{3 S_{1}} \rightarrow \pi^{-}(p n)_{3} S_{1}$. The solid, dashed and dot-dashed lines correspond to nucleons, to pions and to the deuteron (an infinite sum of nucleon bubbles), respectively. All diagrams except (a) and (c1) are one-particle irreducible.

emerges in the diagram Fig. 6b. Note that the corrections to this contribution due to the initial- and finite-state interactions vanish in HP EFT. Here, $\overline{\mathcal{A}(\mu)}$ stands for a sum of all diagrams that do not contain $f_{0}^{r}(\mu)$, see Fig. 6 . At the order we are working, this quantity depends only on the couplings in the two-particle sector $d_{ \pm}, c_{1}$, as well as the masses $M_{\pi}, m$.

The power counting in the expansion parameter $x$ is organized as follows. One groups the diagrams shown in Fig. 6, according to the number $\left(d_{N}\right)$ of virtual $N N$ interactions - "squeezing" all deuteron propagators to a single point. For example, the 1PI diagrams (c2), (c3) correspond to $d_{N}=0,(\mathrm{~d} 1),(\mathrm{d} 2)-$ to $d_{N}=1,(\mathrm{e} 1),(\mathrm{e} 2),(\mathrm{e} 3),(\mathrm{e} 4)-$ to $d_{N}=2$, (f1) - to $d_{N}=3,(\mathrm{~g} 1)-$ to $d_{N}=4$, etc.

For illustration, let us first consider the 1PI diagrams with $d_{N}=0$. The contribution to the threshold amplitude, which is obtained after subtracting all singular pieces from these diagrams, is proportional to powers of the $\pi N$ coupling constants $d_{ \pm}$and depends on the masses $M_{\pi}, m$ and the scale $\mu$. Neither of these quantities scale with $x$ and thus this contribution emerges at $O(1)$.

Next, we consider the diagrams with $d_{N}=1$. The diagram (d1) is proportional to $c_{1} d_{ \pm}^{2}=O\left(x^{-1}\right)$. In addition, there is an intrinsic scale $x$ present in the deuteron propagator $\left(1-c_{1} J\right)^{-1}$, since $c_{1}=O\left(x^{-1}\right)$. In order to get rid of this scale, one has to first perform the contour integration over the time-component of the loop momentum $k^{0}$ and afterwards rescale the loop three-momentum $\mathbf{k} \rightarrow x \mathbf{k}$, 
as well as all external three-momenta. Further, after subtracting all singular pieces at threshold, one may put the external momenta to zero. The counting of the powers of $x$ proceeds as follows. The integration measure $d^{3} \mathbf{k}$ yields the factor $x^{3}$. Since the integration over $k^{0}$ reduces the total number of elementary (pion and nucleon) propagators from three to two and since each elementary propagator counts like $x^{-2}$, this leads to the factor $x^{-4}$ after rescaling the momenta. Finally, taking into account the fact that after rescaling the deuteron propagator counts like $O(1)$ and putting together all factors, we arrive at the conclusion that the diagram (d1) counts like $x^{-2}$ (modulo logarithms). By using the same method, it is easy to show that the diagram $(\mathrm{d} 2)$ contributes at $O\left(x^{-1}\right)$.

Applying the same argument to all diagrams in Fig. 6, we finally come to the conclusion that the leading order scaling of the loop diagrams is that at $O\left(x^{-2}\right)$. Only the diagrams (d1), (e1), (e3), (e4), (f1) contribute at the leading order in $x$. All other diagrams, as well as the diagrams which can be obtained from the diagrams displayed in Fig. 6 by attaching more pion and/or nucleon loops, contribute at a higher order in $x$.

Finally one has to consider the LEC $f_{0}^{r}(\mu)$ which, as we know, scales at $O\left(x^{-2}\right)$ at leading order. Generally, one can write

$$
\begin{aligned}
f_{0}^{r}(\mu) & =F_{-2} x^{-2}+F_{-1} x^{-1}+F_{0}+F_{1} x+\cdots \\
F_{i} & =F_{i}\left(M_{\pi}, m, \mu\right)
\end{aligned}
$$

where only the leading coefficient $F_{-2}$ contributes in the pion-deuteron scattering length at $O(x)$.

To summarize, the right-hand-side of Eq. (4.1) can be written as a sum of the terms that scale as $x^{n}$ with $n=$ $-2,-1,0, \cdots$. The leading-order contribution at $O\left(x^{-2}\right)$ emerges from the diagrams (b), (d1), (e1), (e3), (e4) and (f1). Except the LEC $f_{0}$ that enters from diagram (b), all other diagrams are expressed in terms of the parameters determined in the two-particle sector.

\subsection{Weinberg-Tomozawa term}

Next, we consider the evaluation of the left-hand side of Eq. (4.1). The lowest-order contribution in ChPT in the Weinberg framework emerges from the diagram depicted in Fig. 7. This diagram describes the double scattering of the pion on the nucleons, with the vertices obtained from Weinberg-Tomozawa Lagrangian

$$
\begin{aligned}
\mathcal{L} & =\frac{i}{2 \sqrt{2} F^{2}}\left\{\left(\pi^{+} \partial_{\mu} \pi^{0}-\pi^{0} \partial_{\mu} \pi^{+}\right) \bar{p} \gamma^{\mu} n\right. \\
& \left.+\left(\pi^{-} \partial_{\mu} \pi^{0}-\pi^{0} \partial_{\mu} \pi^{-}\right) \bar{n} \gamma^{\mu} p\right\} \\
& +\frac{i}{4 F^{2}}\left(\pi^{-} \partial_{\mu} \pi^{+}-\pi^{+} \partial_{\mu} \pi^{-}\right)\left\{\bar{p} \gamma^{\mu} p-\bar{n} \gamma^{\mu} n\right\}
\end{aligned}
$$

where one has used the Condon-Shortley convention for the component fields and $F$ stands for the pion decay constant in the chiral limit. At the order of accuracy we

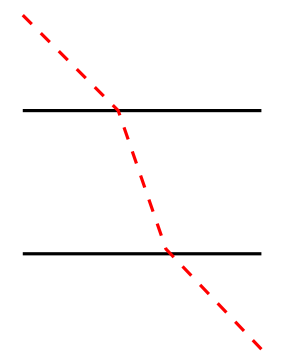

Fig. 7. Lowest-order contribution to the threshold amplitude of the process $\pi^{-} p n \rightarrow \pi^{-} p n$ in ChPT. The solid and dashed lines denote nucleons and pions, respectively.

are working, we may take $F=F_{\pi}=92.4 \mathrm{MeV}$. The scattering amplitude for the process $p\left(q_{1}\right) n\left(q_{2}\right) \pi^{-}\left(q_{3}\right) \rightarrow$ $p\left(p_{1}\right) n\left(p_{2}\right) \pi^{-}\left(p_{3}\right)$ at second order in perturbation theory is given by

$$
\begin{aligned}
T_{W} & =-\frac{1}{4 F^{4}} \frac{\bar{u}\left(p_{1}\right) \not q_{3} u\left(q_{1}\right) \bar{u}\left(p_{2}\right) \not p_{3} u\left(q_{2}\right)}{M_{\pi}^{2}-\left(p_{1}-q_{1}-q_{3}\right)^{2}} \\
& -\frac{1}{4 F^{4}} \frac{\bar{u}\left(p_{1}\right) \not p_{3} u\left(q_{1}\right) \bar{u}\left(p_{2}\right) \not q_{3} u\left(q_{2}\right)}{M_{\pi}^{2}-\left(p_{1}-q_{1}+p_{3}\right)^{2}} \\
& -\frac{1}{2 F^{4}} \frac{\bar{u}\left(p_{1}\right) \not p_{3} u\left(q_{2}\right) \bar{u}\left(p_{2}\right) \not q_{3} u\left(q_{1}\right)}{M_{\pi}^{2}-\left(p_{1}-q_{2}+p_{3}\right)^{2}}
\end{aligned}
$$

The low-energy reduction of the relativistic amplitude Eq. (4.6) yields both the reducible and irreducible parts. In order to single out the 1PI piece, one has to split the relativistic pion propagator into the positive- and negativeenergy components and expand the result for small threemomenta. For example,

$$
\begin{aligned}
& \frac{1}{M_{\pi}^{2}-\left(p_{1}-q_{1}-q_{3}\right)^{2}} \\
= & \frac{1}{2 M_{\pi}} \frac{1}{M_{\pi}-q_{1}^{0}-q_{3}^{0}+p_{1}^{0}+\left(\mathbf{q}_{1}+\mathbf{q}_{3}-\mathbf{p}_{1}\right)^{2} / 2 M_{\pi}}+\cdots \\
+ & \frac{1}{4 M_{\pi}^{2}}+\cdots,
\end{aligned}
$$

where the first (the second) term correspond to the oneparticle reducible (irreducible) pieces. Evaluating the 1PI part at threshold, we get

$$
T_{W}^{(1 \mathrm{PI})}=-\frac{m^{2}}{2 F^{4}}\left(\chi_{1}^{\dagger} \chi_{1} \chi_{2}^{\dagger} \chi_{2}+\chi_{1}^{\dagger} \chi_{2} \chi_{2}^{\dagger} \chi_{1}\right)
$$

where the Pauli spinors are defined through $\sqrt{2 m}\left(\begin{array}{c}\chi_{i} \\ 0\end{array}\right)=$ $\lim _{q_{i} \rightarrow 0} u\left(q_{i}\right)$ and $\sqrt{2 m}\left(\chi_{i}^{\dagger}, 0\right)=\lim _{p_{i} \rightarrow 0} \bar{u}\left(p_{i}\right)$. Performing a Fierz transformation, we get

$$
T_{W}^{(1 \mathrm{PI})}=-\frac{m^{2}}{F^{4}}\left(\chi P_{i} \chi\right)^{\dagger}\left(\chi P_{i} \chi\right), \quad \chi=\left(\begin{array}{c}
\chi_{1} \\
\chi_{2}
\end{array}\right) .
$$

Finally, dividing the amplitude $T_{W}$ by a kinematical factor $(2 m)^{2} 2 M_{\pi}$ that emerges from Eq. (4.1) at threshold, one 
may read off the value of the LEC $f_{0}$, which is obtained through the matching to ChPT at the leading order in chiral expansion

$$
f_{0}=-\frac{1}{8 F^{4} M_{\pi}}+\cdots
$$

Substituting now this value into Eq. (3.22), one ends up with the tiny contribution $-0.0005 M_{\pi}^{-1}$ (cf. with the current experimental value $\left.a_{\pi d}=0.0261 M_{\pi}^{-1}\right)$. The small magnitude for this correction is in agreement with findings of Ref. [14]. The reason why the leading-order chiral contribution is so small is simple: it emerges only at the $\mathrm{NNLO}$ in the $x$ counting and is contained in the term $F_{0}$ of Eq. (4.4). To the contrary, some of the sub-leading contributions in ChPT, which are contained in the $\mathrm{LO}$ and NLO coefficients $F_{-2}, F_{-1}$, get enhanced by inverse powers of $x$ and are numerically much larger that the WeinbergTomozawa term. The above example clearly shows that the power-counting in the HP EFT and in the Weinberg picture are not correlated. In accordance with the findings of Ref. [20], the counting which is based on the expansion parameter $x$, better reflects the numerical size of the contributions emerging at different orders.

\subsection{Initial- and final-state interactions}

From the discussion in the previous section one concludes that one needs to identify a sub-class of the diagrams in the Weinberg approach which - after the matching contributes at $O\left(x^{-2}\right)$ to the LEC $f_{0}$. Since here we are only interested in establishing accuracy limits in the calculations of the pion-deuteron scattering length, in the matching condition we may neglect loop diagrams shown in Fig. 6 and their counterparts in ChPT. Since these diagrams depend only on the parameters that can be determined in the two-particle sector, including these diagrams will only shift the central value of $f_{0}$ determined through matching, without significantly affecting the error bars.

The contributions which one needs to retain in the left-hand side of the matching condition Eq. (4.1), contain the genuine 3-particle ChPT LEC(s) $f_{0}^{\prime}$, which are the counterpart(s) of the LEC $f_{0}$ in HP EFT. Note that due to chiral symmetry, the LECs in the Weinberg framework appear first at $O\left(p^{2}\right)$ - the corresponding Lagrangian should contain either two derivatives of the pion field or the quark mass matrix. Further, these LECs do not come alone: in the spirit of the Weinberg approach, one has in addition to consider summing up the diagrams that correspond to the initial- and final- state interactions of pions and nucleons. Due to the use of the cutoff in the Weinberg approach, this effect does not vanish at threshold, in difference to HP EFT.

In the following, we shall first provide a very crude estimate of the initial- and final-state interaction effect. We assume that the bulk of the effect in the 3-particle system comes from the $N N$ pair, whereas the "hopping" of the pion on the nucleon lines amounts to a small correction. We further approximate the one-pion exchange in

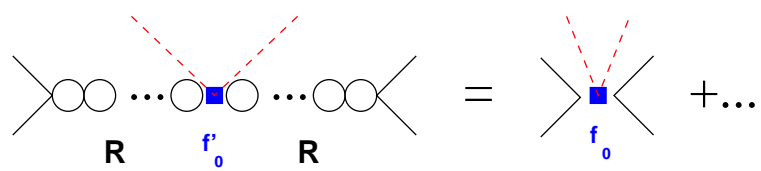

Fig. 8. Including the initial- and final-state interactions in the Weinberg picture

the Weinberg picture by a local $N N$ potential with a coupling denoted by $c_{1}^{\prime}$, assuming a cutoff between the pion mass $M_{\pi}$ and the hard scale $\Lambda$ in the loops, wherever this vertex is present. With these assumptions, the matching condition takes the form that is schematically shown in Fig. 8 (in this figure, the ellipses stand for the diagrams which do not contain 3-particle LECs). One may introduce the "amplification factor" which is obtained by summing up all bubbles in the ingoing and outgoing $N N$ lines

$$
\begin{aligned}
R^{2} & =\frac{1}{\left(1-c_{1}^{\prime}(\Lambda) J_{\Lambda}(0)\right)^{2}}, \\
J_{\Lambda}(|\mathbf{p}|) & =\int^{\Lambda} \frac{d^{3} \mathbf{l}}{(2 \pi)^{3}} \frac{m}{\mathbf{l}^{2}-\mathbf{p}^{2}-i 0} \\
& =\frac{m \Lambda}{2 \pi^{2}}+i \frac{m|\mathbf{p}|}{4 \pi}+O\left(\Lambda^{-1}\right),
\end{aligned}
$$

where we have made explicit the $\Lambda$-dependence of the coupling constant in the Weinberg scheme.

Imposing now the condition that the denominator has a pole at the deuteron binding energy $|\mathbf{p}|=i \gamma$, one can determine the coupling constant $c_{1}^{\prime}(\Lambda)$ and the amplification factor

$$
\begin{aligned}
& \left(c_{1}^{\prime}(\Lambda)\right)^{-1}=\frac{m \Lambda}{2 \pi^{2}}-\frac{m \gamma}{4 \pi}+\cdots, \\
& R^{2}=\left(1-\frac{2 \Lambda}{\pi \gamma}\right)^{2}+\cdots \approx \frac{4 \Lambda^{2}}{\pi^{2} m \epsilon}+\cdots,
\end{aligned}
$$

and the matching gives

$$
f_{0} \propto R^{2} f_{0}^{\prime}+\cdots=r^{2} x^{-2} f_{0}^{\prime}+\cdots,
$$

where $r^{2}$ does not scale with $x$. Hence, the desired scale behavior in the constant $f_{0}$ emerges from the sub-class of the diagrams in the Weinberg picture which describe $N N$ rescattering in the initial and final states in all orders.

The numerical value of the amplification factor $R^{2}$ turns out to be very large: $R^{2} \simeq 4$ for $\Lambda=M_{\pi}, R^{2} \simeq$ 50 for $\Lambda=500 \mathrm{MeV}$ and $R^{2} \simeq 200$ for $\Lambda=1 \mathrm{GeV}$. Performing now a standard dimensional analysis of the LECs in ChPT (see e.g. [39]), we get the estimate of the uncertainty in the quantity $f_{0}$

$$
\Delta f_{0}=\frac{1}{2 M_{\pi}} R^{2} \Delta f_{0}^{\prime}=\frac{1}{2 F_{\pi}^{4} M_{\pi}}\left(\frac{M_{\pi}}{4 \pi F_{\pi}}\right)^{2} R^{2} .
$$

Substituting this result into Eq. (3.22), one finally gets

$$
\begin{aligned}
& \Delta a_{\pi d} / a_{\pi d}=[0.5 \% ; 6 \% ; 23 \%] \\
& \text { for } \Lambda=\left[M_{\pi} ; 500 \mathrm{MeV} ; 1 \mathrm{GeV}\right] .
\end{aligned}
$$


Now we present the exact numerical results in the Weinberg picture which confirm the validity of our crude estimate. Note that, if the calculations are done in the Weinberg approach $a b$ initio, the amplification factor is contained in the quantity $\left|\tilde{\Phi}_{0}(\Lambda)\right|^{2}$, where $\tilde{\Phi}_{0}(\Lambda)$ denotes the value of the wave function at the origin. The counterpart of this quantity in HP EFT is given by $\Phi_{0}=\left(\gamma^{3} / 2 \pi\right)^{\frac{1}{2}}=$ $0.0445 \mathrm{fm}^{-\frac{3}{2}}$. For comparison, the NNLO wave functions in the Weinberg approach are given by

$\tilde{\Phi}_{0}(\Lambda)=[0.487 ; 0.434] \mathrm{fm}^{-\frac{3}{2}}$ for $\Lambda=[450 ; 650] \mathrm{MeV}[40]$. Hence, one gets the following estimate for the amplification factor $R^{2}=\tilde{\Phi}_{0}(\Lambda)^{2} / \Phi_{0}^{2} \simeq 100$, which in turn corresponds to the reasonable value of the cutoff mass $\Lambda \simeq$ $720 \mathrm{MeV}$ in our order-of-magnitude estimate in Eq. (4.15) and to $\Delta a_{\pi d} / a_{\pi d} \simeq 12 \%$, which qualitatively agrees with the large uncertainty in Fig. 5. Note that the $N N$ interactions are now studied up to and including $\mathrm{N}^{3} \mathrm{LO}$ [41]. In our estimates we however use the NNLO wave functions, in order to consistently compare with earlier work on the subject [20].

The LEC $f_{0}$ that enters the expression of the piondeuteron scattering length, can be estimated by using resonance saturation. The details of this procedure can be found in appendix C. It turns out that using the set of parameters determined in Ref. [42] leads to the result which is consistent with the above dimensional estimate. However, due to the large uncertainty in the values of these parameters, the resonance saturation hypothesis can not be used - at present stage - to get a better accuracy in the calculation of $a_{\pi d}$. To this end, one has to improve the quality of the fit of the parameters of the resonance Lagrangian to the data.

How can this large uncertainty be reconciled with the mild cutoff dependence of Ref. [20]? The answer to this question is the following. Assuming for instance that the cutoff dependence of the generic LEC(s) $f_{0}^{\prime}$ in ChPT at the lowest order is logarithmic, we may split these LEC(s) into a term which depends of the cutoff mass $\Lambda$ and a term which does not

$$
f_{0}^{\prime}=A+B \ln \frac{\Lambda}{\Lambda^{*}},
$$

where $\Lambda^{*}$ stands for a some characteristic hard scale (typically $\sim 1 \mathrm{GeV})$. The mild cutoff dependence is equivalent to the statement that the constant $B$ is small. One may argue that the smallness of $B$ is related to the dominance of the one-pion exchange which at the large distances has a softer behavior than the contact 4 -fermion vertex which is used in HP EFT to describe the deuteron. However, the constant $A$ is not covered by above argument. Using the dimensional arguments, we see that the bulk of the uncertainty $\sim 12 \%$ should come from the scale-independent constant $A$.

\section{Comparison to the existing approaches}

The issue of the pion-deuteron scattering has obtained an extensive coverage in the literature during the last decades. A highly incomplete list of references is given in [5-13], see also references therein. Note that the framework used in HP EFT is in fact very close to that of the potential scattering theory. Consequently, a rapid convergence of the series for $a_{\pi d}$ in HP EFT would indicate also on the applicability of the potential picture in the $\pi d$ scattering at threshold. The main question related to choice of the potential remains however open. As was demonstrated in Ref. [43], the conventional quantum-mechanical potentials can be interpreted as a mere regularization of the non-relativistic effective field theories. From this point of view, the scale dependence which was discovered in this article is equivalent to the off-shell effects in the two-body potentials which must be canceled by the corresponding three-body force (analog of the LEC $f_{0}$ ). It is clear that fitting two-body potentials to the scattering data can not completely eliminate this inherent off-shell (scale) dependence. Another aspect of the problem concerns the absence of the relation to QCD and to ChPT. One may conclude that the existing potential models which are used to extract $\pi N$ scattering lengths from the measured $\pi d$ scattering length can not in principle provide enough accuracy needed for the test of the predictions of QCD at low energy in the $\pi N$ sector.

Next, we shall consider the "hybrid" approach in nuclear physics, which is based on the calculation in ChPT of a certain set of Feynman diagrams, corresponding to the "irreducible transition kernel" and finally sandwiching the result by "realistic" wave functions which are calculated, using Paris, Bonn, Argonne,... potentials (see e.g. $[12,14,16])$. As we already mentioned above, this procedure can be justified, if and only if the long-range effects (e.g. the one-pion exchange) dominate the transition operator. However, as we have seen, the situation in describing $\pi d$ scattering length is just the opposite: this quantity receives a large short-distance contribution from the LEC $f_{0}$, which should be there in order to provide a scaleindependence of the final result. In the final expression for the decay width, this constant is multiplied by the wave function of the deuteron at the origin squared. Consequently, for the consistency of the hybrid approach, one must be sure that the value of the "realistic" wave function at the origin is a good approximation of the same quantity, obtained in the effective theory which was previously used to calculate the transition kernel.

A fully consistent approach to the pion-deuteron scattering problem is provided by the effective field theories, in which the wave function of the deuteron is evaluated within the same setting as the diagrams, describing the irreducible kernel. These are e.g. the calculations carried out within the framework with perturbative pions [18], HP EFT with elementary deuteron field [19], or in the Weinberg scheme $[17,20]$. The large scale dependence in the LECs analogous to our $f_{0}$, was first reported in Ref. [18]. The Feynman diagram which leads to such a large dependence is the counterpart of our Fig. 4a. One should however note that treating the pions relativistically and using the chiral Lagrangians unnecessarily complicates the simple physical picture, since the question about the 
convergence of ChPT expansion naturally arises. A more straightforward approach is provided by HP EFT [19]. The authors of this article also find the logarithmic enhancement of the diagram $4 \mathrm{a}$, as well as the large scale dependence which emerges due to this diagram. Note however, that in this paper not all terms at $O(x)$ have taken into account: The diagrams shown in Fig. 4b,c are omitted although, as we have seen, the inclusion of these diagrams does not change the qualitative conclusions. In the present paper, we have also critically re-examined the conjecture made in Ref. [19], concerning the possibility of calculation of the LEC $f_{0}$ with an improved accuracy in the Weinberg picture - this, as was shown, is not possible.

Finally, we briefly comment on the papers $[17,20]$, which provide the systematic treatment of the $\pi d$ scattering problem within the Weinberg framework. The results of these papers are now very easy to understand and the relation to the HP EFT becomes crystal clear. Thus, the puzzle concerning the (seeming) differences between the HP EFT and the Weinberg approach has finally been resolved.

\section{Conclusions}

i) In this paper, we systematically investigate pionic deuterium within the framework of effective field theories. The whole treatment naturally falls into several steps. At the first step, we discuss the extraction of the $\pi d$ scattering length from the $3 p-1 s$ transition energy and width in the pionic deuterium. The next-to-leading order result has been obtained for the level energies, which can be used for an accurate determination of the $\pi d$ threshold scattering amplitude from experimental measurements. Since the isospin-breaking corrections in this amplitude are not expected to be relevant, given the relatively large theoretical uncertainty in connecting $a_{\pi d}$ with the $\pi N$ scattering lengths, these corrections have been neglected for the time being.

ii) The main focus in the present paper is on investigating the possibility to relate $a_{\pi d}$ to the $\pi N$ scattering lengths and on the analysis of the systematic theoretical error in such a procedure. We give a consistent treatment of the problem within the framework of HP $\mathrm{EFT}$, where the expansion parameter is given by the quantity $x=\gamma / M_{\pi} \simeq 1 / 3$, where $\gamma \simeq 45 \mathrm{MeV}$ is the characteristic bound-state momentum in the deuteron. In this paper, we have evaluated contributions to the quantity $a_{\pi d}$, up to and including $O(x)$ that corresponds to the next-to-leading order in HP EFT.

iii) At next-to-leading order, the $\pi d$ scattering length receives a contribution from the (unknown) LEC which we denote as $f_{0}$. We have used the scale-dependence of this contribution for estimating the theoretical error in our calculations. This scale dependence is shown in Fig. 5. The scale must be chosen to be of order of the pion mass, but is otherwise arbitrary. In our opinion, the range $100 \mathrm{MeV}<\mu<250 \mathrm{MeV}$ can be roughly considered as a "natural" choice of this scale. It should be also taken into account that the plot in this figure, which corresponds to the recent experimental measurements of the pionic hydrogen decay width, still does not include the isospin-breaking corrections from ChPT $[28,44]$. As we see from Fig. 5, the theoretical uncertainty due to the unknown LEC $f_{0}$ is rather large.

iv) In this paper, we have investigated in detail the differences between the Weinberg approach and the HP EFT. It was shown that, despite the very mild cutoff dependence in the Weinberg approach, the uncertainty due to the unknown LECs is significant and is of the same order of magnitude as in the HP EFT. The reason for this is that the large initial- and final-state $N N$ interactions lead to the amplification of the initially small LEC contribution. Taking into account this amplification, the theoretical predictions within both approaches are essentially the same.

v) Our main conclusion, concerning the accuracy limits in the extraction of the $\pi N$ scattering lengths from the pion-deuteron data, can be formulated as follows: by far the largest source of uncertainty is the low-energy constant $f_{0}$, which is the genuine short-distance threebody contribution and should be either determined by other experiments or should be obtained by lattice simulations. In particular, one might attempt to get at least the order-of-magnitude estimate from the process $N N \rightarrow N N \pi \pi$ or from the pion-nucleus optical potential, in case of non-equal proton and neutron densities, where $f_{0}$ should be present apart from the "conventional" terms as given in e.g. [45]. Neither of these methods seems easy to be applied. But, without having fixed the value of $f_{0}$ at a sufficient precision, it is impossible to improve the accuracy of the prediction of the pion-deuteron scattering length.

vi) In our opinion, it is feasible to estimate $f_{0}$ by using the resonance saturation hypothesis (see appendix C). At present time, however, the parameters of the resonance Lagrangian are not known at a sufficient precision, and more effort is needed to pin them down accurately from the experimental data.

Acknowledgments: The authors would like to thank V. Baru, S. Beane, B. Borasoy, J. Gasser, D. Gotta, C. Hanhart, H. Krebs, A. Kudryavtsev, B. Metsch, E. Oset, L. Platter, M. Savage and A. Sibirtsev for interesting discussions.

\section{A Calculation of the energy shift of pionic deuterium by using non-relativistic Lagrangians}

In this appendix, we shall present the calculation of the energy of the levels of the pionic deuterium, characterized by the quantum numbers $n l j$. The calculations will be performed up to and including next-to-leading order in isospin-breaking parameters $\alpha$ and $m_{d}-m_{u}$. Since similar calculations for other hadronic bound states have been already considered in the literature in great detail (see e.g. 


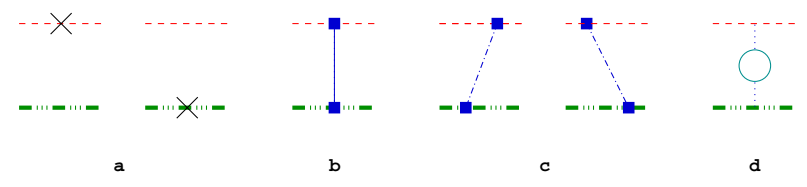

Fig. 9. The contributions to the electromagnetic energy of the pionic deuterium: a) relativistic insertions; b) one Coulomb photon exchange; c) one transverse photon exchange (shaded squares denote pion and deuteron electromagnetic formfactors); d) vacuum polarization.

[23-29]), we shall not repeat them here and display only the most important results. For convenience, the energy of the generic level can be split into the "electromagnetic" and "strong" parts

$$
\begin{aligned}
E_{n l j} & =E_{n}^{\mathrm{C}}+\Delta E_{n l j}^{\mathrm{em}}+\Delta E_{n l j}^{\mathrm{str}}+o\left(\alpha^{4}, \alpha^{3}\left(m_{d}-m_{u}\right)\right), \\
E_{n}^{\mathrm{C}} & =-\frac{1}{2 n^{2}} \alpha^{2} \mu_{d},
\end{aligned}
$$

where $E_{n}^{\mathrm{C}}$ stands for the non-relativistic Coulomb binding energy.

The contributions to the "electromagnetic" part of the potential are depicted in Fig. 9. These include: the relativistic insertions $\mathbf{p}^{4} / 8 M_{\pi}^{3}, \mathbf{p}^{4} / 8 M_{d}^{3}$ in the pion and deuteron lines, respectively; the one (Coulomb and transverse) photon exchange between the pion and the deuteron; the vacuum polarization contribution. At the end of the day, the explicit expression for the potential at this order can be written as

$$
\begin{aligned}
& V_{a b}^{\mathrm{em}}(\mathbf{p}, \mathbf{q})=(2 \pi)^{3} \delta^{3}(\mathbf{q}-\mathbf{p}) \delta_{a b}\left(\frac{\mathbf{p}^{4}}{8 M_{\pi}^{3}}+\frac{\mathbf{p}^{4}}{8 M_{d}^{3}}\right) \\
& +J_{a b}^{0}(\mathbf{p}, \mathbf{q}) \frac{1}{\mathbf{k}^{2}} j^{0}(\mathbf{p}, \mathbf{q}) \\
& +J_{a b}^{\alpha}(\mathbf{p}, \mathbf{q}) \frac{1}{\mathbf{k}^{2}}\left(\delta_{\alpha \beta}-\frac{k_{\alpha} k_{\beta}}{\mathbf{k}^{2}}\right) j^{\beta}(\mathbf{p}, \mathbf{q}) \\
& +\delta_{a b} V^{\mathrm{vac}}(\mathbf{p}, \mathbf{q})
\end{aligned}
$$

where $\mathbf{k}=\mathbf{p}-\mathbf{q}$ and $a, b=1,2,3$ denote the polarization projection of the deuteron. Further, $V^{\text {vac }}$ stands for the vacuum polarization contribution and $J_{a b}^{\mu}, j^{\mu}$ where $\mu=$ $0, \alpha$ denote the electromagnetic formfactor of the deuteron and the pion, respectively. At the order of accuracy we are working, one may use the following expression for these formfactors

$$
\begin{aligned}
J_{a b}^{0}(\mathbf{p}, \mathbf{q}) & =e\left\{\delta_{a b}\left(1-\frac{1}{6}\left\langle r_{d}^{2}\right\rangle \mathbf{k}^{2}\right)+\frac{\mu_{Q}}{2}\left(k_{a} k_{b}-\frac{1}{3} \mathbf{k}^{2} \delta_{a b}\right)\right. \\
& \left.+\frac{1}{4 M_{d}^{2}}\left(Q_{a} k_{b}-k_{a} Q_{b}\right)\left(1-\bar{\mu}_{M}\right)\right\}+O\left(\frac{1}{M_{d}^{3}}\right), \\
J_{a b}^{\alpha}(\mathbf{p}, \mathbf{q}) & =\frac{e}{2 M_{d}}\left\{Q^{\alpha} \delta_{a b}+\bar{\mu}_{M}\left(\delta_{a}^{\alpha} k_{b}-k_{a} \delta_{b}^{\alpha}\right)\right\}+O\left(\frac{1}{M_{d}^{2}}\right),
\end{aligned}
$$

$$
\begin{aligned}
& j^{0}(\mathbf{p}, \mathbf{q})=-e\left(1-\frac{1}{6}\left\langle r_{\pi}^{2}\right\rangle \mathbf{k}^{2}\right)+O\left(\frac{1}{M_{\pi}^{3}}\right), \\
& j^{\alpha}(\mathbf{p}, \mathbf{q})=-\frac{e}{2 M_{\pi}} Q^{\alpha}+O\left(\frac{1}{M_{\pi}^{2}}\right),
\end{aligned}
$$

where $\mathbf{Q}=\mathbf{p}+\mathbf{q}$, and $\mu_{Q}=0.2859 \mathrm{fm}^{2}, \mu_{M}=$ $\frac{e}{2 M_{d}} \bar{\mu}_{M}=0.85741 \frac{e}{2 m}$ are the deuteron quadrupole and magnetic moments, respectively. Substituting these relations in Eq. (A2), we finally obtain

$$
\begin{aligned}
V_{a b}^{\mathrm{em}}(\mathbf{p}, \mathbf{q}) & =-\frac{e^{2}}{\mathbf{k}^{2}} \delta_{a b}+\delta V_{a b}^{\mathrm{em}}(\mathbf{p}, \mathbf{q}) \\
\delta V_{a b}^{\mathrm{em}}(\mathbf{p}, \mathbf{q}) & =(2 \pi)^{3} \delta^{3}(\mathbf{q}-\mathbf{p}) \delta_{a b}\left(\frac{\mathbf{p}^{4}}{8 M_{\pi}^{3}}+\frac{\mathbf{p}^{4}}{8 M_{d}^{3}}\right) \\
& -\frac{e^{2}}{\mathbf{k}^{2}}\left\{-\frac{\mathbf{k}^{2}}{6} \delta_{a b}\left(\left\langle r_{d}^{2}\right\rangle+\left\langle r_{\pi}^{2}\right\rangle\right)\right. \\
& +\frac{1}{4 M_{d} M_{\pi}}\left(\mathbf{Q}^{2}-\frac{(\mathbf{Q} \cdot \mathbf{k})^{2}}{\mathbf{k}^{\mathbf{2}}}\right) \delta_{a b} \\
& +\frac{\mu_{Q}}{2}\left(k_{a} k_{b}-\frac{1}{3} \mathbf{k}^{2} \delta_{a b}\right) \\
& \left.+\left(\frac{1-\bar{\mu}_{M}}{4 M_{d}^{2}}+\frac{\bar{\mu}_{M}}{2 M_{d} M_{\pi}}\right)\left(Q_{a} k_{b}-k_{a} Q_{b}\right)\right\} \\
& +\delta_{a b} V^{\mathrm{vac}}(\mathbf{p}, \mathbf{q}) .
\end{aligned}
$$

The electromagnetic energy shift at next-to-leading order is given by

$$
\begin{aligned}
\Delta E_{n l j}^{\mathrm{em}} & =\sum_{\sigma \rho= \pm, 0} \int \frac{d^{3} \mathbf{p}}{(2 \pi)^{3}} \frac{d^{3} \mathbf{q}}{(2 \pi)^{3}}\langle j \nu \mid l(\nu-\sigma) 1 \sigma\rangle \\
& \times\langle j \nu \mid l(\nu-\rho) 1 \rho\rangle Y_{l(\nu-\sigma)}^{*}(\mathbf{p}) \chi_{a}^{*}(\sigma) \Psi_{n l}^{*}(|\mathbf{p}|) \\
& \times \delta V_{a b}^{\mathrm{em}}(\mathbf{p}, \mathbf{q}) \Psi_{n l}(|\mathbf{q}|) \chi_{b}(\rho) Y_{l(\nu-\rho)}(\mathbf{q}),
\end{aligned}
$$

where $Y_{l \nu}$ and $\langle j m \mid l(m-\sigma) 1 \sigma\rangle$ are the spherical functions and the Clebsch-Gordan coefficients, respectively and

$$
\chi(+)=\frac{1}{\sqrt{2}}\left(\begin{array}{c}
-1 \\
-i \\
0
\end{array}\right), \chi(-)=\frac{1}{\sqrt{2}}\left(\begin{array}{c}
1 \\
-i \\
0
\end{array}\right), \chi(0)=\left(\begin{array}{l}
0 \\
0 \\
1
\end{array}\right)
$$

Note that the energy shift (A5) does not depend on the magnetic quantum number $\nu$. Substituting here the expression (A4), and calculating the integral, we finally obtain

$$
\begin{aligned}
\Delta E_{n l j}^{\mathrm{em}} & =-\frac{M_{d}^{3}+M_{\pi}^{3}}{8 M_{d}^{3} M_{\pi}^{3}}\left(\frac{\alpha \mu_{d}}{n}\right)^{4}\left\{\frac{4 n}{l+1 / 2}-3\right\} \\
& +\delta_{l 0} \frac{2}{3 n^{3}} \alpha^{4} \mu_{d}^{3}\left(\left\langle r_{d}^{2}\right\rangle+\left\langle r_{\pi}^{2}\right\rangle\right)
\end{aligned}
$$




$$
\begin{aligned}
& -\frac{\alpha^{4} \mu_{d}^{3}}{4 M_{d} M_{\pi} n^{4}}\left\{-4 n \delta_{l 0}-4+\frac{6 n}{l+1 / 2}\right\} \\
& +\Delta E_{n l j}^{\mathrm{Q}}+\Delta E_{n l j}^{\mathrm{M}}+\Delta E_{n l j}^{\mathrm{vac}},
\end{aligned}
$$

where for $l \neq 0$

$$
\begin{aligned}
\Delta E_{n l j}^{Q} & =(-1)^{(l+j+1)} \frac{2 \alpha^{4} \mu_{d}^{3}}{n^{3}} \mu_{Q} \sqrt{\frac{30(2 l-2) !}{(2 l+3) !}} \\
& \times\left\{\begin{array}{lll}
l & 1 & j \\
1 & l & 2
\end{array}\right\}, \\
\Delta E_{n l j}^{M} & =(-1)^{(l+j)} \frac{\alpha^{4} \mu_{d}^{3}}{n^{3}} 2 \sqrt{6}\left(\frac{1-\bar{\mu}_{M}}{2 M_{d}^{2}}+\frac{\bar{\mu}_{M}}{M_{d} M_{\pi}}\right) \\
& \times \frac{\left\{\begin{array}{lll}
l & 1 & l \\
1 & j & 1
\end{array}\right\}}{\sqrt{l(l+1)(2 l+1)}},
\end{aligned}
$$

and for $l=0, \Delta E_{n l j}^{Q}=\Delta E_{n l j}^{M}=0$. The quantities in the braces denote the Wigner $6 j$ symbols. Finally, the vacuum polarization contribution is given in Eq. (3) of Ref. [31]. We do not display it here.

Taking $l=1$, we have

$$
\begin{gathered}
\Delta E_{n 1 j}^{Q}=\frac{\alpha^{4} \mu_{d}^{3} \mu_{Q}}{3 n^{3}}\left(\begin{array}{r}
1 ; j=0 \\
-\frac{1}{2} ; j=1 \\
\frac{1}{10} ; j=2
\end{array}\right) \quad \text { (A10) } \\
=\frac{0.015 \mathrm{eV}}{n^{3}}\left(\begin{array}{r}
1 ; j=0 \\
-\frac{1}{2} ; j=1 \\
\frac{1}{10} ; j=2
\end{array}\right) \quad \text { (A11) } \\
\Delta E_{n 1 j}^{M}=\frac{2 \alpha^{4} \mu_{d}^{3}}{3 n^{3}}\left(\frac{1-\bar{\mu}_{M}}{2 M_{d}^{2}}+\frac{\bar{\mu}_{M}}{M_{d} M_{\pi}}\right)\left(\begin{array}{r}
1 ; j=0 \\
\frac{1}{2} ; j=1 \\
-\frac{1}{2} ; j=2
\end{array}\right) \\
=\frac{0.027 \mathrm{eV}}{n^{3}}\left(\begin{array}{r}
1 ; j=0 \\
\frac{1}{2} ; j=1 \\
-\frac{1}{2} ; j=2
\end{array}\right)
\end{gathered}
$$

As we see, the splitting of the energy levels is tiny. Note that in the averaged level energy, defined by Eq. (2.1), these contributions vanish: $\overline{\Delta E_{n l j}^{Q}}=\overline{\Delta E_{n l j}^{M}}=0$. The remaining contributions can be rearranged in order to allow the comparison with the results existing in the literature. In this manner, we finally arrive at the result given in Eqs. $(2.2)$ and (2.3).

\section{B Table of integrals}

After integrating over the 0 th components $l^{0}, k^{0}$, the quantity $R_{a}$ in Eq. (3.22) can be rewritten as

$$
\begin{aligned}
R_{a} & =\int \frac{d^{d} \mathbf{l}}{(2 \pi)^{d}} \frac{d^{d} \mathbf{k}}{(2 \pi)^{d}} \frac{2 m^{3}\left(d_{+}^{2}-2 d_{-}^{2}\right)}{\left(\gamma^{2}+\mathbf{l}^{2}\right)\left(\gamma^{2}+\mathbf{k}^{2}\right)} \\
& \times \frac{1}{\gamma^{2}+\frac{1}{2}\left[\mathbf{l}^{2}+\mathbf{k}^{2}+\frac{m}{M_{\pi}}(\mathbf{l}-\mathbf{k})^{2}\right]} .
\end{aligned}
$$

Introducing Feynman parameters and carrying out the integration over the momenta in the standard manner, one gets

$$
\begin{aligned}
R_{a} & =2 m^{3}\left(d_{+}^{2}-2 d_{-}^{2}\right) \frac{\Gamma(3-d) \mu^{2(d-3)}}{(4 \pi)^{d}}\left(\frac{\gamma^{2}}{\mu^{2}}\right)^{d-3} \\
& \times \int d x_{1} d x_{2} d x_{3} \delta\left(1-x_{1}-x_{2}-x_{3}\right)\left[G_{a}\right]^{-\frac{d}{2}} \\
G_{a} & =x_{1} x_{2}+\frac{x_{3}}{2}\left(1-\frac{x_{3}}{2}+\frac{m}{M_{\pi}}\right),
\end{aligned}
$$

where $\mu$ denotes the scale of dimensional regularization. Renormalizing $R_{a}$ according to the $\overline{\mathrm{MS}}$ prescription, for the finite part we get

$$
\begin{aligned}
R_{a}^{\mathrm{fin}} & =\frac{m^{3}\left(d_{+}^{2}-2 d_{-}^{2}\right)}{32 \pi^{3}}\left(x_{a} \ln \frac{\gamma^{2}}{\mu^{2}}+J_{a}\right)+O(d-3), \\
x_{a} & =-\int d x_{1} d x_{2} d x_{3} \delta\left(1-x_{1}-x_{2}-x_{3}\right)\left[G_{a}\right]^{-\frac{3}{2}} \\
& =-\frac{8 M_{\pi}}{m} \arcsin \frac{m}{m+M_{\pi}}=-1.2569 \cdots, \\
J_{a} & =\frac{1}{2} \int d x_{1} d x_{2} d x_{3} \delta\left(1-x_{1}-x_{2}-x_{3}\right)\left[G_{a}\right]^{-\frac{3}{2}} \ln \left[G_{a}\right] \\
& =-1.1334 \cdots .
\end{aligned}
$$

In the same manner, one obtains

$$
\begin{aligned}
R_{b} & =2 m^{3}\left(d_{+}^{2}+2 d_{-}^{2}\right) \frac{\Gamma(3-d) \mu^{2(d-3)}}{(4 \pi)^{d}} \\
& \times \int_{0}^{1} d x(1-x)\left(\frac{x m}{2 \mu_{N}}\right)^{-\frac{d}{2}}\left(1-\frac{\mu_{N} x}{2 m}\right)^{-\frac{d}{2}},
\end{aligned}
$$

where $\mu_{N}=m M_{\pi}\left(m+M_{\pi}\right)^{-1}$ is the reduced mass in the $\pi N$ system. After the renormalization one may evaluate the remaining integrals analytically. As the result, one obtains

$$
\begin{aligned}
R_{b}^{\mathrm{fin}} & =\frac{m^{3}\left(d_{+}^{2}+2 d_{-}^{2}\right)}{32 \pi^{3}}\left(x_{b} \ln \frac{\gamma^{2}}{\mu^{2}}+J_{b}\right)+O(d-3), \\
x_{b} & =32 \kappa^{\frac{3}{2}} \sqrt{1-\kappa}=0.5098+\cdots \\
J_{b} & =16 \kappa^{\frac{3}{2}}\left\{\frac{2}{\sqrt{\kappa}}(1-2 \kappa) \arcsin \sqrt{\kappa}\right.
\end{aligned}
$$




$$
\begin{aligned}
& -\sqrt{1-\kappa}(2+\ln (1-\kappa)-\ln 4 \kappa)\} \\
& =-0.3731+\cdots, \quad \kappa=\frac{\mu_{N}}{2 m} .
\end{aligned}
$$

The contribution $R_{c}$ can be evaluated in a similar manner. We do not display this calculation here, since this contribution is proportional to $a_{+}^{2}$ and drops anyway. The same method can be applied to the calculation of the diagrams contributing to the threshold scattering amplitude $\mathcal{A}(\mu)$.

\section{Resonance saturation for the LEC $f_{0}$}

In order to get an estimate for the LEC $f_{0}^{\prime}$, we have calculated the tree amplitude of the process $\pi N N \rightarrow \pi N N$ in the theory with the explicit $\Delta, N^{*}(1440)$, scalar and vector mesons in the limit when masses of all above resonances become very large. At threshold, the $\Delta$ and the vector meson do not contribute ${ }^{2}$. In Fig. 10 we display an example of a diagram that does not vanish at threshold. One has 4 such diagrams (insertion of $N^{*}(1440)$ in each nucleon leg), as well as the diagrams which are obtained by a permutation of the outgoing nucleon legs.

The Lagrangian describing the interactions between nucleons, pions, scalar mesons and the $N^{*}(1440)$ is given by (see also [47])

$$
\begin{aligned}
\mathcal{L}^{*} & =g_{s N N} S \bar{N} N+\left[g_{s N N} S \bar{N}^{*} N+\text { h.c. }\right] \\
& +\left[c_{1}^{*} \bar{N}^{*} \chi_{+} N-\frac{c_{2}^{*}}{m^{* 2}}\left(\partial_{\mu} \partial_{\nu} \bar{N}^{*}\right) u^{\mu} u^{\nu} N+\text { h.c. }\right]
\end{aligned}
$$

where $\chi_{+}=M_{\pi}^{2}\left(2-\pi^{2} / F^{2}+\cdots\right)$ and $u_{\mu}=-\boldsymbol{\tau} \partial_{\mu} \boldsymbol{\pi} / F+$ $\cdots$, and $m^{*}$ denotes the mass of the $N^{*}(1440)$. The contribution to the scattering amplitude at threshold in the pertinent spin-isospin channel from the diagram in Fig. 10 is given by

$$
\begin{aligned}
T^{*} & =-(2 m)^{2} \frac{2 M_{\pi}^{2}\left(c_{1}^{*}-c_{2}^{*}\right) g_{s N N} g_{s N N^{*}}}{F^{2} m^{*} M_{S}^{2}} \\
& \times\left(\chi_{1}^{\dagger} \chi_{1} \chi_{2}^{\dagger} \chi_{2}+\chi_{1}^{\dagger} \chi_{2} \chi_{2}^{\dagger} \chi_{1}\right),
\end{aligned}
$$

where $M_{S}$ stands for the mass of the scalar meson. From the above expression, one may read off the contribution to the LEC $f_{0}^{\prime}$

$$
\begin{aligned}
& f_{0}^{\prime}=\frac{\xi}{2 F^{4} M_{\pi}}\left(\frac{M_{\pi}}{4 \pi F}\right)^{2} \\
& \xi=-\frac{64 \pi^{2} F^{4}\left(c_{1}^{*}-c_{2}^{*}\right) g_{s N N} g_{s N N^{*}}}{m^{*} M_{S}^{2}},
\end{aligned}
$$

where, according to the natural size arguments (see Eq. (4.14)), the quantity $\xi$ must be of order 1 .

In the numerical estimates, we use the scalar meson mass $M_{S}=550 \mathrm{MeV}$ (as it was also done in Ref. [47]). Further, $g_{s N N}$ and $g_{s N N^{*}}$ denote the values of the pertinent

\footnotetext{
${ }^{2}$ Vector mesons are described by antisymmetric tensor fields.
}

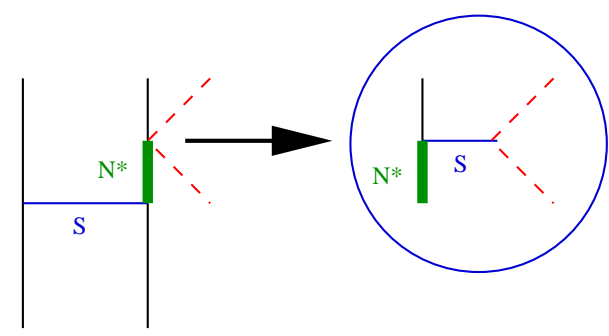

Fig. 10. Tree-level contribution to the scattering amplitude $\pi N N \rightarrow \pi N N$ with the exchange of the $N^{*}(1440)$ and the scalar meson.

formfactors at zero momentum transfer. With the choice of monopole formfactor $F_{S}\left(q^{2}\right)=\left(\Lambda_{S}^{2}-M_{S}^{2}\right) /\left(\Lambda_{S}^{2}-q^{2}\right)$ with $\Lambda_{S}=1700 \mathrm{MeV}$ for both $S N N$ and $S N N^{*}$ vertices, we deduce $g_{s N N}=7.57$ and $g_{s N N^{*}}=3.66$ from the massshell values given e.g. in Ref. [48]. Further, in the same paper, one finds two different values for the constants $c_{1}^{*}, c_{2}^{*}$

$$
\begin{array}{lll}
c_{1}^{*}=-7.27 \mathrm{GeV}^{-1}, & c_{2}^{*}=0 & {[\text { Set 1] },} \\
c_{1}^{*}=-12.7 \mathrm{GeV}^{-1}, & c_{1}^{*}=1.98 \mathrm{GeV}^{-1} & {[\text { Set 2] } .}
\end{array}
$$

Substituting these values, we get $\xi=21$ [Set 1] and $\xi=$ 43 [Set 2] that obviously contradicts to our natural-size estimate. Note that the values for $c_{1}^{*}, c_{2}^{*}$ given in Ref. [48] also do not agree with the result of the fit $c_{1}^{*}+c_{2}^{*}=$ $(-1.56 \pm 3.35) \mathrm{GeV}^{-1}$ from Ref. [42]. However, in order to use the latter fit in our estimate, needs one more relation between $c_{1}^{*}$ and $c_{2}^{*}$. We get this relation, assuming that these constants are saturated by the scalar meson exchange. The Lagrangian describing the interaction of pions with the scalar field with total isospin $I=0$ can be taken from Ref. [49]

$$
\mathcal{L}_{S}=\tilde{c}_{d} S \operatorname{Tr}\left(u_{\mu} u^{\mu}\right)+\tilde{c}_{m} S \operatorname{Tr}\left(\chi_{+}\right) .
$$

from the saturation hypothesis one gets $c_{1}^{*} / c_{2}^{*}=\tilde{c}_{m} / \tilde{c}_{d}=$ $4.2 / 3.2$ (in the last equation, we have used numerical values from Ref. [49]). Together with the value of $c_{1}^{*}+c_{2}^{*}$ from Ref. [42] this yields the estimate $\xi=0.6 \pm 1.3$, which is in a reasonable agreement with the natural-size conjecture. Note that, albeit the resonance saturation method at present does not lead to an improved accuracy as compared to the dimensional analysis, the above discussion demonstrates the feasibility of estimating $f_{0}^{\prime}$ and eventually $f_{0}$ through resonance saturation. What is needed to this end is to determine the LECs $c_{1}^{*}, c_{2}^{*}$ more accurately from the fit to $N^{*}(1440) \rightarrow N \pi \pi$ and, in particular, to resolve the large discrepancies reported in Refs. [42, 48].

\section{References}

1. D. Chatellard et al., Nucl. Phys. A 625 (1997) 855.

2. P. Hauser et al., Phys. Rev. C 58 (1998) 1869.

3. S. Deser, M. L. Goldberger, K. Baumann and W. Thirring, Phys. Rev. 96 (1954) 774.

4. H. C. Schröder et al., Eur. Phys. J. C 21 (2001) 473. 
5. I. R. Afnan and A. W. Thomas, Phys. Rev. C 10 (1974) 109.

6. G. Fäldt, Phys. Scripta 16 (1977) 81.

7. T. Mizutani and D. S. Koltun, Annals Phys. 109 (1977) 1.

8. A. W. Thomas and R. H. Landau, Phys. Rept. 58 (1980) 121.

9. A. Deloff, Phys. Rev. C 64 (2001) 065205 [arXiv:nuclth/0104067].

10. V. V. Baru and A. E. Kudryavtsev, Phys. Atom. Nucl. 60 (1997) 1475 [Yad. Fiz. 60 (1997) 1620]; V. V. Baru, A. E. Kudryavtsev and V. E. Tarasov, Phys. Atom. Nucl. 67 (2004) 743 [Yad. Fiz. 67 (2004) 764] [arXiv:nuclth/0301021].

11. T. E. O. Ericson, B. Loiseau and A. W. Thomas, Phys. Rev. C 66 (2002) 014005 [arXiv:hep-ph/0009312].

12. V. Baru, C. Hanhart, A. E. Kudryavtsev and U.-G. Meißner, Phys. Lett. B 589 (2004) 118 [arXiv:nucl-th/0402027].

13. G. Fäldt and C. Wilkin, arXiv:quant-ph/9610032; A. Boudard, G. Fäldt and C. Wilkin, Phys. Lett. B 389 (1996) 440 [arXiv:nucl-th/9609032].

14. S. Weinberg, Phys. Lett. B 295 (1992) 114 [arXiv:hep$\mathrm{ph} / 9209257]$.

15. N. Kaiser, Phys. Rev. C 65 (2002) 057001 [arXiv:nuclth/0203001].

16. M. Döring, E. Oset and M. J. Vicente Vacas, Phys. Rev. C 70 (2004) 045203 [arXiv:nucl-th/0402086].

17. S. R. Beane, V. Bernard, T.-S. H. Lee and U.-G. Meißner, Phys. Rev. C 57 (1998) 424 [arXiv:nucl-th/9708035].

18. B. Borasoy and H. W. Grießhammer, Int. J. Mod. Phys. E 12 (2003) 65 [arXiv:nucl-th/0105048].

19. S. R. Beane and M. J. Savage, Nucl. Phys. A 717 (2003) 104 [arXiv:nucl-th/0204046].

20. S. R. Beane, V. Bernard, E. Epelbaum, U.-G. Meißner and D. R. Phillips, Nucl. Phys. A 720 (2003) 399 [arXiv:hep$\mathrm{ph} / 0206219]$.

21. H. Krebs, V. Bernard and U.-G. Meißner, Ann. Phys. 316 (2005) 160 [arXiv:nucl-th/0407078].

22. B. F. Irgaziev and B. A. Fayzullaev, arXiv:hep-ph/0404203.

23. A. Gall, J. Gasser, V. E. Lyubovitskij and A. Rusetsky, Phys. Lett. B 462 (1999) 335 [arXiv:hep-ph/9905309].

24. J. Gasser, V. E. Lyubovitskij and A. Rusetsky, Phys. Lett. B 471 (1999) 244 [arXiv:hep-ph/9910438].

25. J. Gasser, V. E. Lyubovitskij, A. Rusetsky and A. Gall, Phys. Rev. D 64 (2001) 016008 [arXiv:hep-ph/0103157].

26. J. Schweizer, Phys. Lett. B 587 (2004) 33 [arXiv:hepph/0401048]; Eur. Phys. J. C 36 (2004) 483 [arXiv:hep$\mathrm{ph} / 0405034$.

27. V. E. Lyubovitskij and A. Rusetsky, Phys. Lett. B 494 (2000) 9 [arXiv:hep-ph/0009206];

28. P. Zemp, in Proceedings of "HadAtom03" Workshop, 13-17 October 2003, ECT* (Trento, Italy), arXiv:hepph/0401204; J. Gasser and P. Zemp, in preparation.

29. U.-G. Meißner, U. Raha and A. Rusetsky, Eur. Phys. J. C 35 (2004) 349 [arXiv:hep-ph/0402261].

30. D. Sigg, A. Badertscher, P. F. A. Goudsmit, H. J. Leisi and G. C. Oades, Nucl. Phys. A 609 (1996) 310.

31. D. Eiras and J. Soto, Phys. Lett. B 491 (2000) 101 [arXiv:hep-ph/0005066].

32. T. E. O. Ericson, B. Loiseau and S. Wycech, Phys. Lett. B 594 (2004) 76.
33. J. Bijnens and P. Talavera, JHEP 0203 (2002) 046 [arXiv:hep-ph/0203049].

34. F. Schmidt-Kaler, D. Leibfried, M. Weitz, and T. W. Hänsch, Phys. Rev. Lett. 70 (1993) 2261.

35. U.-G. Meißner and S. Steininger, Phys. Lett. B 419 (1998) 403 [arXiv:hep-ph/9709453]; G. Müller and U.-G. Meißner, Nucl. Phys. B 556 (1999) 265 [arXiv:hep-ph/9903375]; N. Fettes and U.-G. Meißner, Nucl. Phys. A 693 (2001) 693 [arXiv:hep-ph/0101030].

36. J. Gasser, M. A. Ivanov, E. Lipartia, M. Mojzis and A. Rusetsky, Eur. Phys. J. C 26 (2002) 13 [arXiv:hepph/0206068].

37. U. van Kolck, UMI-94-01021; U. van Kolck, Few Body Syst. Suppl. 9 (1995) 444; E. Epelbaum and U.-G. Meißner, Phys. Lett. B 461 (1999) 287 [arXiv:nucl-th/9902042]; J. L. Friar and U. van Kolck, Phys. Rev. C 60 (1999) 034006 [arXiv:nucl-th/9906048]. M. Walzl, U.-G. Meißner and E. Epelbaum, Nucl. Phys. A 693 (2001) 663

[arXiv:nucl-th/0010019]; E. Epelbaum, U.-G. Meißner and J. E. Palomar, arXiv:nucl-th/0407037, Phys. Rev. C (in print). J. L. Friar, G. L. Payne and U. van Kolck, arXiv:nucl-th/0408033, Phys. Rev. C (in print).

38. D. Gotta, in proceedings of the 8th International Workshop on Meson Production, Properties and Interaction, Krakow, Poland, 4-8 June 2004, International Journal of Modern Physics A (in print).

39. J. L. Friar, Few Body Syst. 22 (1997) 161 [arXiv:nuclth/9607020].

40. We thank H. Krebs for providing us with these values.

41. E. Epelbaum, W. Glöckle and U.-G. Meißner, Nucl. Phys. A 747 (2005) 362 [arXiv:nucl-th/0405048].

42. V. Bernard, N. Kaiser and U.-G. Meißner, Nucl. Phys. B 457 (1995) 147 [arXiv:hep-ph/9507418].

43. E. Lipartia, V. E. Lyubovitskij and A. Rusetsky, Phys. Lett. B 533 (2002) 285 [arXiv:hep-ph/0110186].

44. P. Büttiker et al., in progress.

45. C. Garcia-Recio, E. Oset and L. L. Salcedo, Phys. Rev. C 37 (1988) 194; C. Garcia-Recio, J. Nieves and E. Oset, Nucl. Phys. A 547 (1992) 473.

46. J. Gasser, arXiv:hep-ph/0412393.

47. V. Bernard, N. Kaiser and U.-G. Meißner, Nucl. Phys. A 615 (1997) 483 [arXiv:hep-ph/9611253].

48. L. Alvarez-Ruso, E. Oset and E. Hernandez, Nucl. Phys. A 633 (1998) 519 [arXiv:nucl-th/9706046].

49. G. Ecker, J. Gasser, A. Pich and E. de Rafael, Nucl. Phys. B 321 (1989) 311. 


\section{Erratum}

In the above paper we have studied the pion-deuteron scattering length within low-energy effective theories of QCD. In particular, in the section 4.3 of this article we give an estimate of the uncertainty in this scattering length, coming from the presence of the 6-particle low-energy constant (LEC) - referred to as $f_{0}$. Unfortunately, in this estimate, which has yielded $\Delta a_{\pi d} / a_{\pi d} \simeq 12 \%$, an incorrect value for the deuteron wave function at the origin in the Weinberg approach $\tilde{\Phi}_{0}(\Lambda)=[0.487 ; 0.434] \mathrm{fm}^{-3 / 2}$ with the cutoff mass from the interval $\Lambda=[450 ; 650]$ has been used (normalization error). Using the corrected value $\tilde{\Phi}_{0}(\Lambda)=[0.137 ; 0.122] \mathrm{fm}^{-3 / 2}$ downsizes the error (only due to the above-mentioned source) to a much more comfortable $\Delta a_{\pi d} / a_{\pi d} \simeq 1 \%$, which, in addition, fits better to the a priori estimates, based on the Weinberg power counting. Thus, the results of the high-precision calculations of Ref. [20] have been independently confirmed.

This result has far-reaching consequences. In particular, we have to re-think the equivalence between the Weinberg [20] and HPEFT [18, 19] approaches to the piondeuteron scattering at low energy. The arguments remain the same, but the big change in the estimated uncertainty leads to the conclusion that now this equivalence is realized in a slightly different manner. As before, the success of the modified power counting [20] unambiguously indicates that HPEFT is a physically equivalent tool for describing pion-deuteron interactions near threshold. Furthermore, the sole input which HPEFT imports from the Weinberg approach are the values of LECs, determined through the matching procedure. As it is discussed in the above paper, these LECs in general acquire contributions from two different momentum regions: $M_{\pi}<p<\Lambda$ and $p>\Lambda$. Whereas the former can in principle be expressed through the wave functions, etc - and is thus calculable in terms of the known parameters of the theory, the latter represents a genuinely high-energy contribution, which at the present stage can only be included in the error estimate. What follows from our corrected calculations is that this high-energy contribution is small and does not preclude a high-precision determination of the pion-nucleon scattering lengths from the combined analysis of the pionic hydrogen and pionic deuterium data by Pionic Hydrogen collaboration at PSI $[1,2]$. We wish to emphasize once more that the strong predictive power is a result of a subtle balance between HPEFT, which at the end allows for constructing multiple scattering series in terms of observables (scattering lengths, etc) and the Weinberg approach, which enables one to evaluate the LECs of HPEFT with a high precision.

Several remarks are in order. First, we wish to mention that our error estimate, which is carried out by using dimensional arguments or resonance saturation hypothesis, is complementary to the study of the scale dependence of the calculated pion-deuteron scattering length. Obviously, the investigations should proceed from both ends. As mentioned in the above paper, a weak scale dependence would indicate that the uncertainty might be small and not that it must be small. Finally, the fact that the short-range interactions do not introduce large uncertainty in the calculated value of the pion-deuteron scattering length, might stimulate further activity in calculating various small contributions, with an aim to determine this scattering length as precise as possible. Using the above-mentioned equivalence between the Weinberg and HPEFT approaches could enable one to carry out these calculations in a simpler setting. 